\title{
MODULE CATEGORIES OVER POINTED HOPF ALGEBRAS
}

\author{
MARTÍN MOMBELLI
}

\begin{abstract}
We develop some techniques to the study of exact module categories over some families of pointed finite-dimensional Hopf algebras. As an application we classify exact module categories over the tensor category of representations of the small quantum groups $u_{q}\left(\mathfrak{s l}_{2}\right)$.
\end{abstract}

\section{INTRODUCTION}

Given a tensor category $\mathcal{C}$, a module category over $\mathcal{C}$ is an abelian category $\mathcal{M}$ together with an exact functor $\mathcal{C} \times \mathcal{M} \rightarrow \mathcal{M}$ satisfying natural associativity and unity axioms. This rather general notion appears, and it is implicit, in many different areas of mathematics and mathematical physics; such as the theory of weak Hopf algebras O03a]; subfactor theory [BEK00]; extensions of vertex algebras [KO02]; affine Hecke algebras [BO01] and conformal field theory, see for example [FS01, CS07, CS08. In this case one is interested in the tensor category of representations of a certain vertex algebra, and irreducible objects of a module category are interpreted as boundary conditions for the conformal field theory.

The language of module categories was used with profit in diverse papers. In the study of fusion categories, see [ENO05, ENO08, in relation with dynamical twists M07. In N07 module categories were used to describe some properties of semisimple Hopf algebras.

Etingof and Ostrik proposed [EO04], as an important problem, the classification of a certain class of module categories, called exact, and not only of semisimple module categories. This was done in the framework of the study of finite tensor categories not necessarily semisimple, motivated by its relation, among others, with logarithmic conformal field theories. Such classification is available only for few examples, see [EO04, O03a, O03b], O08.

This paper arises mainly motivated by the problem of classifying module categories over the tensor category of representations of the small quantum groups $u_{q}(\mathfrak{g})$, for a simple Lie algebra $\mathfrak{g}$ and $q$ a primitive root of unity.

Date: February 20, 2019.

2000 Mathematics Subject Classification. 16W30, 18D10, 19D23.

This work was conducted during a post-doc fellowship at the Ludwig Maximilians Universität, München, granted by the Deutscher Akademischer Austausch Dienst (DAAD). 
One of the goals of this paper is the classification in the case $\mathfrak{g}=\mathfrak{s l}_{2}$. This particular problem was proposed by V. Ostrik at the meeting Groupes quantiques dynamiques et categories de fusion, at CIRM, Marseille 2008.

The paper is organized as follows. In section 2 we give a brief account of the basic definitions of graded and coradically graded Hopf algebras, twisting in Hopf algebras and Hopf-Galois extensions. In section 3 we recall some basic facts on exact module categories as introduced by Etingof and Ostrik EO04, and also we recall some results on module categories over the tensor category of representations of a finite-dimensional Hopf algebra. In particular we recall the main result from [AM07] that any exact indecomposable module category over $\operatorname{Rep}(H), H$ a finite-dimensional Hopf algebra, is equivalent to the category of left $K$-modules, for some $H$-simple left $H$-comodule $K$.

Section 4 is devoted to the study of filtered (graded) comodule algebras over a filtered (graded) Hopf algebra. In section 5 we study the relation between liftings of graded comodule algebras, that is comodule algebras $A$ such that gr $A \simeq G$, where $G$ is a given graded comodule algebra, and formal deformations of $G$. This is completely analogous to the study of liftings of Hopf algebras in DCY05.

The different liftings of a given comodule algebras are controlled by a certain double complex which we present in section 6 generalizing previous results of D. Yau [Y07, Y08.

In section 7 we show that if $A$ is a certain graded comodule algebras over a Hopf algebra $H$ constructed by a bosonization as $H=\mathfrak{B}(V) \# \mathbb{k} G$, where $\mathfrak{B}(V)$ is the Nichols algebra of a Yetter-Drinfeld module $V$ over the group algebra $\mathbb{k} G$ over a cyclic group $G$, then $A$ is determined by a certain homogeneous coideal subalgebra in $\mathfrak{B}(V)$ and a subgroup of $G$.

Using the techniques developed previously, in section 8 we present the classification of exact module categories over the Taft Hopf algebras, the Radford Hopf algebras, the book Hopf algebras and over the Lusztig small quantum groups $u_{q}\left(\mathfrak{s l}_{2}\right)$.

\section{PReliminaries And NOtATion}

Throughout this work $\mathbb{k}$ will denote an algebraically close field of characteristic zero. All vector spaces, algebras and linear categories will be consider over $\mathbb{k}$. By $\mathbb{k}^{\times}$we shall denote the non-zero elements of $\mathbb{k}$.

If $A$ is an algebra, we shall denote by ${ }_{A} \mathcal{M}$ the category finite-dimensional left $A$-modules. If $H$ is a Hopf algebra $\operatorname{Rep}(H)$ will denote the tensor category of finite-dimensional representations of $H$ and ${ }_{H}^{H} \mathcal{Y} D$ will denote the category of Yetter-Drinfeld modules of $H$.

Given a Hopf algebra $H$, a left $H$-comodule algebra is a collection $(A, \mu, \lambda)$, where $(A, \mu)$ is an algebra, $\lambda: A \rightarrow H \otimes_{\mathbb{R}} A$ is the $H$-comodule structure 
on $A$ such that $\lambda$ is an algebra map. We shall sometimes denote this comodule algebra by $(A, \lambda)$. We will denote it $(A, \mu, \lambda)$ only when special emphasis on the product is needed. The set of coinvariants of $A$ is $A^{\mathrm{co} H}=\{a \in A: \lambda(a)=1 \otimes a\}$.

If $A$ is an $H$-comodule algebra via $\lambda: A \rightarrow H \otimes_{\mathbb{k}} A$, we shall say that a (right) ideal $J$ is $H$-costable if $\lambda(J) \subseteq H \otimes_{\mathbb{k}} A$. We shall say that $A$ is (right) $H$-simple, if there is no nontrivial (right) ideal in $A, H$-costable.

Let $A$ be a left $H$-comodule algebra. We denote by ${ }^{H} \mathcal{M}_{A}$ the category of left $H$-comodules, right $A$-modules $M$ such that the right $A$-module structure $M \otimes_{\mathbb{k}} A \rightarrow M$ is an $H$-comodule map. If $P \in{ }^{H} \mathcal{M}_{A}$ we will denote by $\operatorname{End}_{A}^{H}(P)$ the space of $A$-module and $H$-comodule endomorphisms.

A left coideal subalgebra of $H$ is a subalgebra $K \subseteq H$ such that $\Delta(K) \subseteq$ $H \otimes_{\mathbb{k}} K$. It is well-known that if $H$ is finite-dimensional, $K$ is a left coideal subalgebra of $H$ and $Q$ denotes the coalgebra $H / H K^{+}$, then the pair of functors $\mathcal{F}:{ }^{H} \mathcal{M}_{K} \rightarrow{ }^{Q} \mathcal{M}, \mathcal{F}(M)=M / M K^{+}$and $\mathcal{G}:{ }^{Q} \mathcal{M} \rightarrow{ }^{H} \mathcal{M}_{K}$, $\mathcal{G}(V)=H \square_{Q} V$ gives an equivalence of categories. See [Ma92], Sk07].

For a coalgebra $C$ we shall denote by $C_{0}$ the coradical and by $C_{0} \subseteq C_{1} \subseteq$ $C_{1} \cdots \subseteq C_{n} \subseteq \ldots$ the coradical filtration. For more details see [Sw69].

Let us recall that a coalgebra $C$ is said to be $\mathbb{N}_{0}$-graded, or graded for short, if $C=\bigoplus_{i} C(i)$, such that $\Delta(C(n)) \subseteq \bigoplus_{i=0}^{n} C(i) \otimes_{\mathbb{k}} C(n-i)$ for all $n \in \mathbb{N}_{0}$ and $\varepsilon(C(n))=0$ for all $n \geq 1$. A coalgebra is coradically graded if it is graded and the coradical filtration is given by $C_{n}=\bigoplus_{i=0}^{n} C(i)$.

A Hopf algebra $H$ is $\mathbb{N}_{0}$-graded if it is $\mathbb{N}_{0}$-graded as an algebra and a coalgebra by the same grading.

A filtration on a Hopf algebra $H$ is an algebra filtration $H^{0} \subseteq H^{1} \subseteq$ $\cdots \subseteq H^{m}=H$ such that for all $n=0 \ldots m$

$$
\Delta\left(H^{n}\right) \subseteq \sum_{i=0}^{n} H^{i} \otimes_{\mathbb{k}} H^{n-i} .
$$

If $H$ is a filtered Hopf algebra such that $H^{0}$ is a Hopf subalgebra then the graded algebra associated to the filtration gr $H=\bigoplus_{n \geq 0} H^{n} / H^{n-1}$, here $H^{-1}=0$, is a graded Hopf algebra. The associated graded Hopf algebra to the coradical filtration will be denoted by $\mathrm{gr}_{c} H$. It is a well-known fact that $\operatorname{gr}_{c} H$ is a coradically graded Hopf algebra.

Let $H$ be a graded Hopf algebra. A lifting of $H$ is filtered Hopf algebra structure $U$ on the same underlying vector space $H$ such that $\operatorname{gr} U=H$.

Two liftings $U$ and $V$ of $H$ are said to be equivalent if there is a Hopf algebra isomorphism $\phi: U \rightarrow V$ such that $\operatorname{gr} \phi=\mathrm{id}{ }_{H}$. The set of equivalence classes of liftings of $H$ will be denoted by $\operatorname{Lift}(H)$. 
2.1. Twisting comodule algebras. Let us recall that a Hopf 2-cocycle is a map $\sigma: H \otimes_{\mathbb{k}} H \rightarrow \mathbb{k}$, invertible with respect to convolution, such that

$$
\begin{aligned}
\sigma\left(x_{(1)}, y_{(1)}\right) \sigma\left(x_{(2)} y_{(2)}, z\right) & =\sigma\left(y_{(1)}, z_{(1)}\right) \sigma\left(x, y_{(2)} z_{(2)}\right), \\
\sigma(x, 1) & =\varepsilon(x)=\sigma(1, x),
\end{aligned}
$$

for all $x, y, z \in H$. Using this cocycle there is a new Hopf algebra structure constructed over the same coalgebra $H$ with the product described by

$$
x_{\cdot[\sigma]} y=\sigma\left(x_{(1)}, y_{(1)}\right) \sigma^{-1}\left(x_{(3)}, y_{(3)}\right) x_{(2)} y_{(2)}, \quad x, y \in H .
$$

We shall denote by $H^{\sigma}$ this new Hopf algebra. We shall denote by ${ }_{\sigma} H$ the space $H$ with new multiplication given by

$$
x \cdot y=\sigma\left(x_{(2)}, y_{(2)}\right) x_{(1)} y_{(1)},
$$

for all $x, y \in H$. The algebra ${ }_{\sigma} H$ is a left $H$-comodule algebra with coaction given by the coproduct of $H$.

We shall say that $\sigma$ is cocentral if for all $x, y \in H$

$$
\sigma\left(x_{(1)}, y_{(1)}\right) x_{(2)} y_{(2)}=\sigma\left(x_{(2)}, y_{(2)}\right) x_{(1)} y_{(1)} .
$$

It is obvious that $\sigma$ is cocentral if and only if $H^{\sigma}=H$. A useful way to check if a certain 2-cocycle $\sigma$ is cocentral is to prove that the coproduct ${ }_{\sigma} H: \rightarrow{ }_{\sigma} H \otimes_{\mathbb{k}} H$ makes ${ }_{\sigma} H$ a right $H$-comodule algebra. This observation will be used later without further mention.

If $\sigma: H \otimes H \rightarrow \mathbb{k}$ is a 2-cocycle and $K$ is a left $H$-comodule algebra, then we can define a new product in $K$ by

$$
a_{\sigma} b=\sigma\left(a_{(-1)}, b_{(-1)}\right) a_{(0)} \cdot b_{(0)},
$$

$a, b \in K$. We shall denote by $K_{\sigma}$ this new algebra. It is easy to see that $K_{\sigma}$ is a left $H^{\sigma}$-comodule algebra. The following basic result will be useful later.

Lemma 2.1. Let $\sigma: H \otimes H \rightarrow \mathbb{k}$ be a Hopf 2-cocycle and $K$ be a left $H$ comodule algebra. There is an equivalence of categories ${ }^{H} \mathcal{M}_{K} \simeq H^{\sigma} \mathcal{M}_{K_{\sigma}}$.

Proof. If $M \in{ }^{H} \mathcal{M}_{K}$ we define a right $K_{\sigma}$-module structure by

$$
m \cdot{ }_{\sigma} k=\sigma\left(m_{(-1)}, k_{(-1)}\right) m_{(0)} \cdot k_{(0)},
$$

for all $k \in K, m \in M$. We shall denote by $M_{\sigma}$ the object $M$ with this new action and the same left $H$-comodule structure. By a straightforward computation one can prove that $M_{\sigma} \in H^{\sigma} \mathcal{M}_{K_{\sigma}}$ and that the functor $M \longmapsto$ $M_{\sigma}$ is an equivalence of categories.

In particular if $K \subseteq H$ is a left coideal subalgebra, $Q=H / H K^{+}$and $\sigma: H \otimes H \rightarrow \mathbb{k}$ is a cocentral 2-cocycle then the categories ${ }^{H} \mathcal{M}_{K_{\sigma}},{ }^{Q} \mathcal{M}$ are equivalent. 
The following definition will be used later.

Definition 2.2. A Hopf 2-cocycle $\sigma: H \otimes H \rightarrow \mathbb{k}$ is compatible with a left coideal subalgebra $K \subseteq H$ if for any $x, y \in K, \sigma\left(x_{(2)}, y_{(2)}\right) x_{(1)} y_{(1)} \in K$.

In the case when $\sigma$ is compatible with $K$ we denote ${ }_{\sigma} K$ the left $H$ comodule algebra obtained from $K$ using multiplication given as in (2.3). Any cocentral 2-cocycle is compatible with any left coideal subalgebra and in this case ${ }_{\sigma} K=K_{\sigma}$.

2.2. Hopf Galois extensions. Let $H$ be a Hopf algebra. A left $H$-Galois extension of an algebra $R$ is an algebra $A$ with a left $H$-comodule algebra structure, $A \rightarrow H \otimes_{\mathbb{k}} A, a \rightarrow a_{(-1)} \otimes a_{(0)}$, such that $A^{\text {co } H}=R$ and the canonical map can $: A \otimes_{R} A \rightarrow H \otimes A, \operatorname{can}(a \otimes b)=a_{(-1)} \otimes a_{(0)} b$ is a bijection. For more details on this subject the reader is referred to [SS04].

It is well known that if $\sigma: H \otimes_{\mathbb{k}} H \rightarrow \mathbb{k}$ is a 2-cocycle then ${ }_{\sigma} H$ is a $H$ Galois extension of the field $\mathbb{k}$. In the finite-dimensional case the converse also holds, that is, if $H$ is finite-dimensional and $A$ is a finite-dimensional left $H$-Galois extension of the field then there is a 2-cocycle $\sigma$ such that $A \simeq{ }_{\sigma} H$.

\section{EXact module CATEgories OVER finite tensor CATEgories}

We recall the definitions of module categories over finite tensor categories. For more details the reader is referred to [O03a, EO04].

A module category over a tensor category $\mathcal{C}$ is an abelian category $\mathcal{M}$ provided with an exact bifunctor $\otimes: \mathcal{C} \times \mathcal{M} \rightarrow \mathcal{M}$ and natural associativity and unit isomorphisms $m_{X, Y, M}:(X \otimes Y) \otimes M \rightarrow X \otimes(Y \otimes M), \ell_{M}: \mathbf{1} \otimes M \rightarrow$ $M$ such that for any $X, Y, Z \in \mathcal{C}, M \in \mathcal{M}$.

$$
\begin{aligned}
\left(\text { id } \otimes m_{Y, Z, M}\right) m_{X, Y \otimes Z, M}\left(a_{X, Y, Z} \otimes i d\right) & =m_{X, Y, Z \otimes M} m_{X \otimes Y, Z, M}, \\
\left(\text { id } \otimes \ell_{M}\right) m_{X, \mathbf{1}, Y} & =r_{X} \otimes \text { id } .
\end{aligned}
$$

A module functor between module categories $\mathcal{M}$ and $\mathcal{M}^{\prime}$ over a tensor category $\mathcal{C}$ is a pair $(\mathcal{F}, c)$, where $\mathcal{F}: \mathcal{M} \rightarrow \mathcal{M}^{\prime}$ is a $\mathbb{k}$-linear functor and $c_{X, M}: \mathcal{F}(X \otimes M) \rightarrow X \otimes \mathcal{F}(M)$ is a natural isomorphism such that for any $X, Y \in \mathcal{C}, M \in \mathcal{M}:$

$$
\begin{aligned}
\left(\operatorname{id}_{X} \otimes c_{Y, M}\right) c_{X, Y \otimes M} \mathcal{F}\left(m_{X, Y, M}\right) & =m_{X, Y, \mathcal{F}(M)} c_{X \otimes Y, M}, \\
\ell_{\mathcal{F}(M)} c_{\mathbf{1}, M} & =\mathcal{F}\left(\ell_{M}\right) .
\end{aligned}
$$

Let $\mathcal{M}_{1}$ and $\mathcal{M}_{2}$ be module categories over $\mathcal{C}$. We shall denote by $\operatorname{Hom}_{\mathcal{C}}\left(\mathcal{M}_{1}, \mathcal{M}_{2}\right)$ the category whose objects are module functors $(\mathcal{F}, c)$ from $\mathcal{M}_{1}$ to $\mathcal{M}_{2}$. A morphism between $(\mathcal{F}, c)$ and $(\mathcal{G}, d) \in \operatorname{Hom}_{\mathcal{C}}\left(\mathcal{M}_{1}, \mathcal{M}_{2}\right)$ is a natural transformation $\alpha: \mathcal{F} \rightarrow \mathcal{G}$ such that for any $X \in \mathcal{C}, M \in \mathcal{M}_{1}$ :

$$
d_{X, M} \alpha_{X \otimes M}=\left(\operatorname{id}_{X} \otimes \alpha_{M}\right) c_{X, M} .
$$


Two module categories $\mathcal{M}_{1}$ and $\mathcal{M}_{2}$ over $\mathcal{C}$ are equivalent if there exist module functors $F: \mathcal{M}_{1} \rightarrow \mathcal{M}_{2}$ and $G: \mathcal{M}_{2} \rightarrow \mathcal{M}_{1}$ and natural isomorphisms id $\mathcal{M}_{1} \rightarrow F \circ G$, id $\mathcal{M}_{2} \rightarrow G \circ F$ that satisfy (3.5).

The direct sum of two module categories $\mathcal{M}_{1}$ and $\mathcal{M}_{2}$ over a tensor category $\mathcal{C}$ is the $\mathbb{k}$-linear category $\mathcal{M}_{1} \times \mathcal{M}_{2}$ with coordinate-wise module structure.

A module category is indecomposable if it is not equivalent to a direct sum of two non trivial module categories.

In this paper we further assume that all module categories have finitely many isomorphism classes of simple objects. The following definition of a very important class of module categories is due to P. Etingof and V. Ostrik.

Definition 3.1. EO04 A module category $\mathcal{M}$ over a finite tensor category $\mathcal{C}$ is exact if for any projective $P \in \mathcal{C}$ and any $M \in \mathcal{M}$, the object $P \otimes M$ is again projective in $\mathcal{M}$.

Any semisimple finite module category over a finite tensor category $\mathcal{C}$ is exact. A direct sum of finite module categories is exact if and only if each summand is exact. Therefore, any exact module category over $\mathcal{C}$ is a finite direct product of exact indecomposable module categories, see EO04, Prop. $3.9]$.

3.1. Module categories over Hopf algebras. We recall some results concerning exact module categories over the category of representations of Hopf algebras. Let $H$ be a finite dimensional Hopf algebra.

If $\lambda: A \rightarrow H \otimes_{\mathbb{k}} A$ is a left $H$-comodule algebra then the category of finite-dimensional left $A$-modules ${ }_{A} \mathcal{M}$ is a module category over $\operatorname{Rep}(H)$ with action $\bar{\otimes}: \operatorname{Rep}(H) \times{ }_{A} \mathcal{M} \rightarrow{ }_{A} \mathcal{M}, X \bar{\otimes} M=X \otimes_{\mathbb{R}} M$, for all $X \in$ $\operatorname{Rep}(H), M \in{ }_{A} \mathcal{M}$. The left $A$-module structure on $X \otimes_{\mathbb{K}} M$ is given by $\lambda$, that is, if $a \in A, x \in X, m \in M$ then $a \cdot(x \otimes m)=a_{(-1)} \cdot x \otimes a_{(0)} \cdot m$.

If $A$ is right $H$-simple then ${ }_{A} \mathcal{M}$ is an exact indecomposable module category over $\operatorname{Rep}(H)$, see [AM07, Prop. 1.20]. The exactness is a consequence of the beautiful results obtained by Skryabin [Sk07] on comodule algebras.

Remark 3.2. In particular the category ${ }_{A} \mathcal{M}$ is an exact indecomposable module category over $\operatorname{Rep}(H)$ when

(1) $A=K_{\sigma}, K \subseteq H$ is a coideal subalgebra and $\sigma: H \otimes_{\mathbb{k}} H \rightarrow \mathbb{k}$ is a cocentral Hopf 2-cocycle,

(2) or, more generally, $A={ }_{\sigma} K$ where $K \subseteq H$ is a left coideal subalgebra and $\sigma: H \otimes_{\mathbb{k}} H \rightarrow \mathbb{k}$ is a 2-cocycle compatible with $K$.

Theorem 3.3. AM07, Theorem 3.3] If $\mathcal{M}$ is an exact idecomposable module category over $\operatorname{Rep}(H)$ then $\mathcal{M} \simeq{ }_{A} \mathcal{M}$ for some $H$-simple left comodule algebra $A$ with $A^{\mathrm{co} H}=\mathbb{k}$. 
Two left $H$-comodule algebras $A$ and $B$ are Morita equivariant equivalent, and we shall denote it by $A \sim_{M} B$, if the module categories ${ }_{A} \mathcal{M},{ }_{B} \mathcal{M}$ are equivalent as module categories over $\operatorname{Rep}(H)$.

Proposition 3.4. AM07, Prop. 1.24] The algebras $A$ and $B$ are Morita equivariant equivalent if and only if there exists $P \in{ }^{H} \mathcal{M}_{B}$ such that $A \simeq$ $\operatorname{End}_{B}(P)$ as $H$-comodule algebras.

The left $H$-comodule structure on $\operatorname{End}_{B}(P)$ is given by $\lambda: \operatorname{End}_{B}(P) \rightarrow$ $H \otimes_{\mathbb{k}} \operatorname{End}_{B}(P), \lambda(T)=T_{(-1)} \otimes T_{(0)}$ where

$$
\left\langle\alpha, T_{(-1)}\right\rangle T_{0}(p)=\left\langle\alpha, T\left(p_{(0)}\right)_{(-1)} \mathcal{S}^{-1}\left(p_{(-1)}\right)\right\rangle T\left(p_{(0)}\right)_{(0)},
$$

for any $\alpha \in H^{*}, T \in \operatorname{End}_{B}(P), p \in P$. It is easy to prove that $\operatorname{End}_{B}(P)^{\text {co } H}=$ $\operatorname{End}_{B}^{H}(P)$.

Let $K \subseteq H$ be a left coideal subalgebra and $\sigma: H \otimes_{\mathbb{k}} H \rightarrow \mathbb{k}$ be a cocentral 2-cocycle. Let us denote by $Q$ the quotient $H / H K^{+}$.

Lemma 3.5. Let $A$ be a $H$-simple left $H$-comodule algebra with trivial coinvariants Morita equivariant equivalent to $K_{\sigma}$. Then there exists an indecomposable object $P \in Q_{\mathcal{M}}$ such that $A \simeq \operatorname{End}_{K_{\sigma}}\left(\left(H \square_{Q} P\right)_{\sigma}\right)$ and $\operatorname{dim} A=(\operatorname{dim} P)^{2} \operatorname{dim} K$.

Proof. The existence of the object $P$ such that $A \simeq \operatorname{End}_{K_{\sigma}}\left(\left(H \square_{Q} P\right)_{\sigma}\right)$ follows by Proposition 3.4 and Lemma 2.1. Since the coinvariants are trivial $A^{\text {co } H}=\mathbb{k}$ then $\operatorname{End}_{B}^{H}\left(H \square_{Q} P\right)=\mathbb{k}$ and this implies that $H \square_{Q} P$ must be indecomposable thus $P$ is also indecomposable. Since $H \simeq K \otimes Q$ as left $K$-modules, right $Q$-comodules, then $H \square_{Q} P \simeq K \otimes P$, whence $\operatorname{dim} A=$ $(\operatorname{dim} P)^{2} \operatorname{dim} K$.

Let us further assume that $Q$ is a pointed cosemisimple coalgebra. Thus, if $P \in{ }^{Q} \mathcal{M}$ is an indecomposable object then is 1-dimensional and there exists a group-like element $g \in Q$ and $0 \neq v \in P$ such that $P=\mathbb{k}<v>$ and $\delta(v)=g \otimes v$ is the left $Q$-comodule structure on $P$. In this case $H \square_{Q} P \simeq g K$.

Lemma 3.6. Under the above assumptions if $A$ is a left $H$-comodule algebra such that $A \sim_{M} K_{\sigma}$ then $A \simeq\left(g K g^{-1}\right)_{\sigma^{g}}$, for some group-like element $g \in G(H)$ and $\sigma^{g}: H \otimes_{\mathbb{k}} H \rightarrow \mathbb{k}$ is the Hopf 2-cocycle given by

$$
\sigma^{g}(x, y)=\sigma\left(g^{-1} x g, g^{-1} y g\right)
$$

for any $x, y \in H$.

Proof. From the above considerations $A \simeq \operatorname{End}_{K_{\sigma}}\left((g K)_{\sigma}\right)$. Any element $T \in \operatorname{End}_{K_{\sigma}}\left((g K)_{\sigma}\right)$ is determined by the value $T(g)$. Indeed, any element $g x \in g K$ can be written uniquely $g x=g \cdot_{\sigma}\left(\sigma^{-1}\left(g, x_{(1)}\right) x_{(2)}\right)$. Here, for any $x, y \in H$ we are denoting $x \cdot \sigma y=\sigma\left(x_{(1)}, y_{(1)}\right) x_{(2)} y_{(2)}$ the product as in (2.5). 
The maps $\phi:\left(g K g^{-1}\right)_{\sigma^{g}} \rightarrow \operatorname{End}_{K_{\sigma}}\left((K g)_{\sigma}\right), \psi: \operatorname{End}_{K_{\sigma}}\left((K g)_{\sigma}\right) \rightarrow$ $\left(g K g^{-1}\right)_{\sigma^{g}}$ given by

$$
\phi\left(g y^{-1} g\right)(g \cdot x)=g \cdot(x \cdot y), \quad \psi(T)=g^{-1} T(g),
$$

for any $x, y \in K T \in \operatorname{End}_{K_{\sigma}}\left((K g)_{\sigma}\right)$ are well-defined maps one the inverse of each other. It is straightforward to verify that both maps are $H$-comodule morphisms.

Example 3.7. Let $G$ be a finite group, $F \subseteq G$ be a subgroup and $H=\mathbb{k} G$ is the group algebra. The group algebra $\mathbb{k} F$ is a left coideal subalgebra of $H$. Let also $\psi \in Z^{2}\left(G, \mathbb{k}^{\times}\right)$be a (necessarily cocentral) 2-cocycle. Let us denote by $\mathbb{k}^{\psi} F$ the twisted group algebra. An indecomposable object $P \in Q_{\mathcal{M}}$, $Q=\mathbb{k} G /(\mathbb{k} F)^{+} \mathbb{k} G$ must be one-dimensional, say $P=<\mathbb{k} \bar{g}>$. If $A \sim_{M} \mathbb{k}^{\psi} F$ then there exists an element $g \in G$ such that $A \simeq \mathbb{k}^{\psi^{g}} F^{g}$, where $F^{g}$ is the conjugate of $F$. This result is contained in [O03a, Thm 2].

\section{Graded And filtered Comodule Algebras}

Let us assume that $H$ is a finite-dimensional filtered Hopf algebra with filtration given by $H^{0} \subseteq H^{1} \subseteq \cdots \subseteq H^{m}=H$ such that $H^{0}$ is a Hopf subalgebra.

Given a left $H$-comodule algebra $(A, \lambda)$, we shall say that a filtration $A^{0} \subseteq A^{1} \subseteq \cdots \subseteq A^{m}$ is a comodule algebra filtration compatible with the filtration of $H$ if it is an algebra filtration and for each $n$

$$
\lambda\left(A^{n}\right) \subseteq \sum_{i=0}^{n} H^{i} \otimes_{\mathbb{R}} A^{n-i} .
$$

Observe that $A^{0}$ is a left $H^{0}$-comodule algebra. Whenever no confusion arises we shall simply say that $A$ is a filtered $H$-comodule algebra.

Any filtration on the Hopf algebra induces a compatible filtration on a comodule algebra.

Lemma 4.1. Let $H^{0} \subseteq H^{1} \subseteq \cdots \subseteq H^{m}=H$ be a filtration on the Hopf algebra $H$ and $(A, \lambda)$ a comodule algebra. Define $A^{n}=\lambda^{-1}\left(H^{n} \otimes A\right)$ for all $n=0 \ldots m$, then $A^{0} \subseteq \cdots \subseteq A^{m}=A$ is a filtration compatible with the filtration on $H$.

Proof. Since $\lambda$ is an algebra map, for any $i, j$ we have that $A^{i} A^{j} \subseteq A^{i+j}$. Let $0 \leq n \leq m$ and $a \in A^{n}$, then $\lambda(a) \in H^{n} \otimes A$, hence

$$
\left(\operatorname{id}_{H} \otimes \lambda\right) \lambda(a)=\left(\Delta \otimes \operatorname{id}_{A}\right) \lambda(a) \in \sum_{i=0}^{n} H^{i} \otimes H^{n-i} \otimes A .
$$

Whence $\lambda(a) \in \sum_{i=0}^{n} H^{i} \otimes A^{n-i}$. 
As a consequence any comodule algebra $(A, \lambda)$ has a distinguished filtration compatible with the coradical filtration on $H$, the Loewy series on $A$; it is the filtration $A_{0} \subseteq A_{1} \subseteq \cdots \subseteq A_{m}$, defined by $A_{n}=\lambda^{-1}\left(H_{n} \otimes A\right)$ for all $n \in \mathbb{N}$.

Definition 4.2. Let $H=\oplus_{i=0}^{m} H(i)$ be a graded Hopf algebra. We shall say that a left $H$-comodule algebra $G$, with comodule structure given by $\lambda: G \rightarrow H \otimes_{\mathbb{k}} G$, graded as an algebra $G=\oplus_{i=0}^{m} G(i)$ is a graded comodule algebra if for each $0 \leq n \leq m$

$$
\lambda(G(n)) \subseteq \bigoplus_{i=0}^{m} H(i) \otimes_{\mathbb{k}} G(n-i) .
$$

A graded comodule algebra $G=\oplus_{i=0}^{m} G(i)$ is Loewy-graded if it is a graded comodule algebra and the Loewy series is given by $G_{n}=\oplus_{i=0}^{n} G(i)$.

Let $(A, \lambda)$ be a filtered left $H$-comodule algebra, $A^{0} \subseteq A^{1} \subseteq \cdots \subseteq A^{m}$ compatible with the filtration $H^{0} \subseteq H^{1} \subseteq \cdots \subseteq H^{m}=H$. We can consider the graded algebra gr $A=\bigoplus_{n \geq 0} A^{n} / A^{n-1}$. The graded algebra associated to the Loewy series will be denoted by $\operatorname{gr}_{l} A$.

There is a well defined map $\bar{\lambda}: \operatorname{gr} A \rightarrow \operatorname{gr} H \otimes \operatorname{gr} A$ such that the following diagram commute

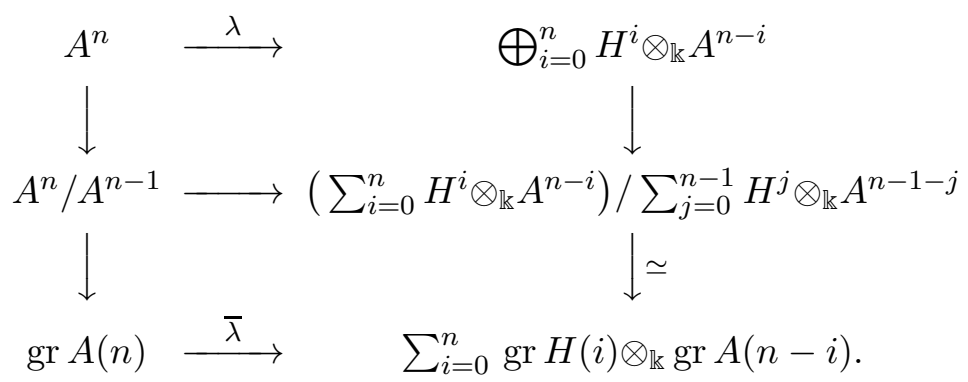

Lemma 4.3. The space $(\operatorname{gr} A, \bar{\lambda})$ is a graded gr $H$-comodule algebra and $\left(\operatorname{gr}_{l} A, \bar{\lambda}\right)$ is a Loewy-graded $\operatorname{gr}_{c} H$-comodule algebra.

Proof. This is a well-known fact. The proof can be found for example in [AD03], Sw69].

The following result will be useful when we relate module categories over a Hopf algebra $H$ and over $\operatorname{gr}_{c} H$.

Proposition 4.4. Let $H^{0} \subseteq H^{1} \subseteq \cdots \subseteq H^{m}=H$ be a filtration on the Hopf algebra $H$ such that $H^{0}$ is a semisimple Hopf subalgebra. Let also $A$ be a left $H$-comodule algebra and consider the filtration $A^{i}=\lambda^{-1}\left(H^{i} \otimes_{\mathbb{k}} A\right)$. The following assertions are equivalent.

(1) $A^{0}$ is right $H^{0}$-simple.

(2) $A$ is right $H$-simple. 
Proof. Let us assume that $A^{0}$ is right $H^{0}$-simple. Let $J \subseteq A$ be right ideal $H$-costable. Consider the filtration on $J ; J^{0} \subseteq J^{1} \subseteq \ldots J^{m}=J$ given by $J^{i}=\lambda^{-1}\left(H^{i} \otimes_{\mathbb{k}} J\right)$. The space $J^{0} \subseteq A^{0}$ is a right ideal $H^{0}$-costable, thus $J^{0}=0$ or $J^{0}=A^{0}$. In the second case $1 \in J$ and thus $J=A$. Let us assume that $J^{0}=0$. Follows from Lemma 4.1 that $\lambda\left(J^{n}\right) \subseteq \sum_{i=0}^{n} H^{i} \otimes_{\mathbb{k}} J^{n-i}$. Then $\lambda\left(J^{n}\right) \subseteq H_{0} \otimes_{\mathbb{k}} J^{1}$ thus $J^{1}=J^{0}$. Arguing inductively follows that $J^{n}=J^{n-1}$ for all $n$, thus $J=0$.

Assume $A$ is right $H$-simple. Since $H^{0}$ is semisimple, using [Li03, Thm. 3.1], we obtain that the Jacobson radical $J\left(A^{0}\right)$ is a $H^{0}$-costable ideal of $A^{0}$, hence $J\left(A^{0}\right) A$ is a right ideal $H$-costable in $A$. Since the radical is nilpotent the equality $J\left(A^{0}\right) A=A$ is impossible, therefore $J\left(A^{0}\right) A=0$ and $A^{0}$ is semisimple. Thus, the inclusion $A^{0} \subseteq A$ splits, that is, there is a left $A^{0}$ module $B \subseteq A$ such that $A=A^{0} \oplus B$. Now, Let $J \subseteq A^{0}$ be a nonzero right $H^{0}$-costable ideal. Then $J A=J \oplus J B \subseteq J \oplus B$. Since $A$ is right $H$-simple we obtain that $J A=A$ which implies that $J=A^{0}$.

Under the same hypothesis as Proposition 4.4 we have the following result.

Corollary 4.5. The following assertions are equivalent.

(1) $A^{0}$ is right $H^{0}$-simple.

(2) gr $A$ is right gr $H$-simple.

(3) $A$ is right $H$-simple.

Proof. (1) is equivalent to (3) is Proposition 4.4. But the same proof works for $\operatorname{gr} A$ since $(\operatorname{gr} A)^{0}=A^{0}$.

\section{LifTingS AND FORMAL DEFORMATION OF COMODULE ALGEBRAS}

For the rest of this section $(H, \Delta, m)$ will denote a graded Hopf algebra with grading $H=\oplus_{i=0}^{m} H(i)$.

Let $G$ be a graded $H$-comodule algebra. If $U$ is a lifting for $H$, we shall say that $A$ is a lifting for $G$ along $U$ if $A=G$ as vector spaces and $A$ is a filtered left $U$-comodule algebra and gr $A \simeq G$ as left $H$-comodule algebras.

Two liftings $A, B$ of $G$ along $U$ are said to be equivalent if there is an isomorphism of filtered $U$-comodule algebras $\psi: A \rightarrow B$ such that gr $\psi=$ id $_{G}$. We shall denote by $\operatorname{Lift}(A, U)$ the set of equivalence classes of liftings of $A$ along $U$.

5.1. Formal bialgebra deformation. Let us recall graded bialgebra deformation from DCY05. See also [MW07. For any $l \in \mathbb{N} \cup\{\infty\}$ consider the free $\mathbb{k}[t] /\left(t^{l+1}\right)$-module $H[t] /\left(t^{l+1}\right)$ as a graded vector space over $\mathbb{k}$ by

$\operatorname{deg} t=1$ and $\operatorname{deg} h=i$ if $h \in H(i)$. If $l=\infty, H[t] /\left(t^{l+1}\right)=H[t]$ and $\mathbb{k}[t] /\left(t^{l+1}\right)=\mathbb{k}[t]$. 
An $l$-deformation of $H$ is a collection $\left(H[t] /\left(t^{l+1}\right), m^{l}, \Delta^{l}\right)$, where

$m^{l}:\left(H \otimes_{\mathbb{k}} H\right)[t] /\left(t^{l+1}\right) \rightarrow H[t] /\left(t^{l+1}\right), \quad \Delta^{l}: H[t] /\left(t^{l+1}\right) \rightarrow\left(H \otimes_{\mathbb{k}} H\right)[t] /\left(t^{l+1}\right)$ are $\mathbb{k}[t] /\left(t^{l+1}\right)$-linear maps homogeneous of degree 0 , such that $H[t] /\left(t^{l+1}\right)$ is a bialgebra. This implies that there are maps $\Delta_{s}^{l}: H \rightarrow H \otimes_{\mathbb{k}} H, m_{s}^{l}$ : $H \otimes_{\mathbb{k}} H \rightarrow H$ of degree $-s$ such that for all $x, y \in H$

$$
\Delta^{l}(x)=\sum_{s=0}^{l} \Delta_{s}^{l}(x) t^{s}, \quad m^{l}(x \otimes y)=\sum_{s=0}^{l} m_{s}^{l}(x \otimes y) t^{s} .
$$

It is also required that $\Delta_{0}^{l}=\Delta$ and $m_{0}^{l}=m$.

Two $l$-deformations $\left(H[t] /\left(t^{l+1}\right), m^{l}, \Delta^{l}\right)$ and $\left(H[t] /\left(t^{l+1}\right), \widetilde{m}^{l}, \widetilde{\Delta}^{l}\right)$ are isomorphic if there exists a bialgebra isomorphism $\phi: H[t] /\left(t^{l+1}\right) \rightarrow H[t] /\left(t^{l+1}\right)$ homogeneous of degree 0 . In this case there are maps $\phi_{s}: H \rightarrow H$ homogeneous of degree $-s$ such that for any $h \in H, \phi(h)=\sum_{s=0}^{l} \phi_{s}(h) t^{s}$. Here it is required that $\phi_{0}=\mathrm{id}{ }_{H}$.

The set of equivalence of $l$-deformations is denoted by $I s o^{l}(H)$. When $l=\infty$, this set is simply denoted by $I s o(H)$.

The following result is [DCY05, Thm. 2.2].

Theorem 5.1. There exists a natural bijection between Lift $(H)$ and Iso( $H)$.

5.2. Formal deformation for comodule algebras. Let us fix a $l$-deformation $\left(H[t] /\left(t^{l+1}\right), m^{l}, \Delta^{l}\right)$ of $H$ that we shall denote by $H_{l}$ for short. Let also $(G, \mu, \lambda)$ be a graded left $H$-comodule algebra.

Definition 5.2. An $l$-deformation of $G$ is a collection $\left(G[t] /\left(t^{l+1}\right), \mu^{l}, \lambda^{l}\right)$ where $\mu^{l}:\left(G \otimes_{\mathbb{k}} G\right)[t] /\left(t^{l+1}\right) \rightarrow G[t] /\left(t^{l+1}\right), \lambda^{l}: G[t] /\left(t^{l+1}\right) \rightarrow\left(H \otimes_{\mathbb{k}} G\right)[t] /\left(t^{l+1}\right)$ are $\mathbb{k}[t] /\left(t^{l+1}\right)$-linear maps homogeneous of degree zero such that $G[t] /\left(t^{l+1}\right)$ is a left $H_{l}$-comodule algebra. In particular there are maps $\lambda_{s}^{l}: G \rightarrow H \otimes_{\mathbb{k}} G$, $\mu_{s}^{l}: G \otimes_{\mathbb{k}} G \rightarrow G$ homogeneous of degree $-s$ such that for any $a, b \in G$

$$
\lambda^{l}(a)=\sum_{s=0}^{l} \lambda_{s}^{l}(a) t^{s}, \quad \mu^{l}(a \otimes b)=\sum_{s=0}^{l} \mu_{s}^{l}(a \otimes b) t^{s} .
$$

It is required that $\lambda_{0}^{l}=\lambda$ and $\mu_{0}^{l}=\mu$.

The associativity of the product, the coassociativity and the compatibility of the coaction of $G[t] /\left(t^{l+1}\right)$ with the product imply that for any $0 \leq n \leq l$

$$
\begin{gathered}
\sum_{i+j=n} \mu_{i}^{l}\left(\mu_{j}^{l} \otimes \mathrm{id}_{G}\right)=\sum_{i+j=n} \mu_{i}^{l}\left(\mathrm{id}_{G} \otimes \mu_{j}^{l}\right), \\
\sum_{i+j=n}\left(\mathrm{id}_{H} \otimes \lambda_{i}^{l}\right) \lambda_{j}^{l}=\sum_{i+j=n}\left(\Delta_{i}^{l} \otimes \mathrm{id}_{G}\right) \lambda_{j}^{l}, \\
\sum_{i+j=n} \lambda_{i}^{l} \mu_{j}^{l}=\sum_{i+j+k+r=n}\left(m_{i}^{l} \otimes \mu_{j}^{l}\right) \tau_{23}\left(\lambda_{k}^{l} \otimes \lambda_{r}^{l}\right) .
\end{gathered}
$$


Two $l$-deformations $\left(G[t] /\left(t^{l+1}\right), \mu^{l}, \lambda^{l}\right),\left(\widetilde{G}[t] /\left(t^{l+1}\right), \widetilde{\mu}^{l}, \widetilde{\lambda}^{l}\right)$ are equivalent if there exists an homogeneous map of degree zero $\phi: G[t] /\left(t^{l+1}\right) \rightarrow$ $\widetilde{G}[t] /\left(t^{l+1}\right)$ such that $\phi$ is a $\mathbb{k}[t] /\left(t^{l+1}\right)$-linear isomorphism of $H_{l}$-comodule algebras. In particular there are homogeneous maps $\phi_{s}: G \rightarrow \widetilde{G}$ of degree $-s$ such that

$$
\phi(a)=\sum_{s=0}^{l} \phi_{s}(a) t^{s},
$$

for all $a \in G$. We required that $\phi_{0}=\mathrm{id}_{G}$. Denote by $\operatorname{Iso}^{l}\left(G, H_{l}\right)$ the set of equivalence classes of $l$-deformations of $G$. The proof of the following Lemma is completely analogous to [DCY05, Lemma 2.1].

Lemma 5.3. For $l>l^{\prime}$ there exist a restriction map $\operatorname{res}_{l, l^{\prime}}: I_{s o}{ }^{l}\left(G, H_{l}\right) \rightarrow$ $I$ so $^{l^{\prime}}\left(G, H_{l^{\prime}}\right)$, and maps $r_{l}: I_{s o}^{\infty}\left(G, H_{\infty}\right) \rightarrow I s o^{l}\left(G, H_{l}\right)$ such that

$$
I s o^{\infty}\left(G, H_{\infty}\right) \simeq \lim _{l \in \mathbb{N}} I \operatorname{so}^{l}\left(G, H_{l}\right)
$$

The result DCY05, Th. 2.2] can be extended to the comodule algebra setting in a natural way. Let $U$ be a lifting of $H$ corresponding to the $l$-deformation $H_{l}$ under Theorem 5.1 .

Theorem 5.4. There is a natural bijection $\operatorname{Iso}^{\infty}\left(G, H_{\infty}\right) \simeq \operatorname{Lift}(G, U)$.

The following technical Lemma will be crucial to find liftings of certain comodule algebras over pointed Hopf algebras.

Let $F$ be a finite group, $H=\oplus_{i=0}^{m} H(i)$ be a finite-dimensional coradically graded Hopf algebra, $U^{0} \subseteq U^{1} \subseteq \cdots \subseteq U^{m}=U$ a filtered Hopf algebra $U$ with $U^{0}=\mathbb{k} F=H(0)$, such that gr $U=H$. Let $g \in F$ and $x \in U^{1}-U^{0}$ an element such that $\Delta(x)=x \otimes 1+g \otimes x$

Let $\left(G, \lambda_{0}\right)$ be a Loewy-graded $H$-comodule with grading $G=\oplus_{i=0}^{m} G(i)$ such that $G(0) \simeq \mathbb{k} \widetilde{F}$, where $\widetilde{F} \subseteq F$ is a subgroup, that is there exists a base $\left\{e_{f}: f \in \widetilde{F}\right\}$ of $G$ such that the product and the comodule structure are given by

$$
e_{f} e_{h}=e_{f h}, \quad \lambda\left(e_{f}\right)=f \otimes e_{f},
$$

for any $f, h \in \widetilde{F}$. Let $(A, \lambda)$ be a left $U$-comodule algebra and let us consider the filtration $A^{i}=\lambda^{-1}\left(U^{i} \otimes_{\mathbb{k}} A\right)$. Assume that gr $A=G$.

Lemma 5.5. Under the above assumptions, if $y \in A^{1}$ is an element such that

$$
\lambda_{0}(\bar{y})=x \otimes 1+g \otimes \bar{y},
$$

then $\lambda(y)=x \otimes 1+g \otimes y$. Here $\bar{y}$ denotes the class of $y$ in $A^{1} / A^{0}=G(1)$.

Proof. Let $y \in A^{1}$ be an element such that $\lambda_{0}(y)=x \otimes 1+g \otimes \bar{y}$. Since we know that there are homogeneous maps $\lambda_{s}: G \rightarrow H \otimes_{\mathbb{k}} G$ of degree $-s$, such that the coaction of $A$ is $\lambda=\sum_{s=0} \lambda_{s}$, then $\lambda(y)=x \otimes 1+g \otimes y+\lambda_{1}(y)$ 
where $\lambda_{1}(y) \in U_{0} \otimes A_{0}$. Set $\lambda_{1}(y)=\sum_{h \in F, f \in \widetilde{F}} \alpha_{h, f} h \otimes e_{f}$, where $\alpha_{h, f} \in \mathbb{k}$. Since $(\operatorname{id} \otimes \lambda) \lambda=(\Delta \otimes$ id $) \lambda$ then

$$
\sum_{h \in F, f \in \widetilde{F}} \alpha_{h, f} h \otimes h \otimes e_{f}=\sum_{h \in F, f \in \widetilde{F}} \alpha_{h, f} h \otimes f \otimes e_{f},
$$

whence we deduce that $\alpha_{h, f}=0$ if $h \neq f$, thus $\lambda_{1}(y)=\sum_{f \in F} \alpha_{f, f} f \otimes e_{f}$. Since $(\varepsilon \otimes$ id $) \lambda(y)=y$ then $(\varepsilon \otimes$ id $) \lambda_{1}(y)=0$, thus

$$
\sum_{f \in \widetilde{F}} \alpha_{f, f}(\varepsilon \otimes \mathrm{id})\left(f \otimes e_{f}\right)=\sum_{f \in \widetilde{F}} \alpha_{f, f} e_{f}=0 .
$$

Therefore $\lambda_{1}(y)=0$.

\section{Deformation BiCOMPleX FOR COMODUle AlgEBRAS}

In Y08 and [Y07] the author introduce a bicomplex to study algebraic deformations of $H$-comodule algebras $A$ over a graded Hopf algebra $H$. The cohomology groups of the total complex are related to formal deformations of $A$ over the same Hopf algebra $H$. In this section we introduce a certain bicomplex generalizing the constructed by D. Yau in a way that cohomology groups of the total complex are related to formal deformations of $A$ over a any lifting $U$ of $H$.

6.1. Bialgebra cohomology. Let $H$ be a bialgebra. For each $s \in \mathbb{N}$ and each $1 \leq i \leq s$ define the maps $m_{i}^{s}, \lambda_{l}^{s}, \lambda_{r}^{s}: H^{s+1} \rightarrow H^{s}$ and $\Delta_{i}^{s}, \delta_{l}^{s}, \delta_{r}^{s}$ : $H^{s} \rightarrow H^{s+1}$ by

$$
\begin{aligned}
m_{i}^{s}\left(y^{1} \otimes \ldots \otimes y^{s+1}\right) & =y^{1} \otimes \ldots \otimes y^{i} y^{i+1} \otimes \ldots \otimes y^{s+1}, \\
\Delta_{i}^{s}\left(y^{1} \otimes \ldots y^{s}\right) & =y^{1} \otimes \ldots \otimes y^{i}{ }_{(1)} \otimes y_{2}^{i} \otimes \ldots \otimes y^{s}, \\
\lambda_{l}^{s}\left(x \otimes y^{1} \otimes \ldots y^{s}\right) & =x_{(1)} y^{1} \otimes \ldots \otimes x_{(s)} y^{s}, \\
\lambda_{r}^{s}\left(y^{1} \otimes \ldots y^{s} \otimes x\right) & =y^{1} x_{(1)} \otimes \ldots \otimes y^{s} x_{(s)}, \\
\delta_{l}^{s}\left(y^{1} \otimes \ldots y^{s}\right) & =y^{1}{ }_{(1)} \ldots y^{s}{ }_{(1)} \otimes y^{1}{ }_{(2)} \otimes \ldots \otimes y_{(2)}^{s}, \\
\delta_{r}^{s}\left(y^{1} \otimes \ldots y^{s}\right) & =y_{(1)}^{1} \otimes \ldots \otimes y_{(1)}^{s} \otimes y_{(2)}{ }_{(2)} \ldots y_{(2)}^{s},
\end{aligned}
$$

for all $x, y^{1}, \ldots, y^{s} \in H$.

Let $C^{p, q}(H)=\operatorname{Hom}_{\mathbb{k}}\left(H^{\otimes p}, H^{\otimes q}\right)$ and let

$$
\partial_{p, q}^{h}: C^{p, q} \rightarrow C^{p+1, q}, \quad \partial_{p, q}^{v}: C^{p, q} \rightarrow C^{p, q+1},
$$

be the maps defined by $\partial_{p, q}^{h}=\sum_{i=0}^{p+1}(-1)^{i} \partial_{p, q}^{h}[i]$, and $\partial_{p, q}^{v}=\sum_{i=0}^{q+1}(-1)^{i} \partial_{p, q}^{v}[i]$, where for any $f \in C^{p, q}(H)$

$$
\partial_{p, q}^{v}[i](f)= \begin{cases}\left(\mathrm{id}_{H} \otimes f\right) \delta_{l}^{p} & \text { if } i=0 \\ \Delta_{i}^{q} \circ f & \text { if } 1 \leq i \leq q \\ \left(f \otimes \mathrm{id}_{H}\right) \delta_{r}^{p} & \text { if } i=q+1\end{cases}
$$

and 


$$
\partial_{p, q}^{h}[i](f)= \begin{cases}\lambda_{l}^{q}\left(\mathrm{id}_{H} \otimes f\right) & \text { if } i=0 \\ f \circ m_{i}^{p} & \text { if } 1 \leq i \leq p \\ \lambda_{r}^{q}\left(f \otimes \mathrm{id}_{H}\right) & \text { if } i=p+1\end{cases}
$$

We will use the truncated complex, that is $C^{p, q}(H)=0$ if $p q=0$.

6.2. Deformation bicomplex for comodule algebras. Let $(A, \mu, \lambda)$ be a left $H$-comodule. For any $n \in \mathbb{N}$ and $a^{i} \in A$ define the maps

$$
\lambda_{1}^{n}: A^{\otimes n} \rightarrow H \otimes_{\mathbb{k}} A^{\otimes n}, \quad \lambda_{2}^{n}: A^{\otimes n} \rightarrow H^{\otimes n} \otimes_{\mathbb{k}} A
$$

by

$$
\begin{aligned}
& \lambda_{1}^{n}\left(a^{1} \otimes \ldots \otimes a^{n}\right)=a^{1}{ }_{(-1)} \ldots a^{n}{ }_{(-1)} \otimes a^{1}{ }_{(0)} \otimes \ldots \otimes a^{n}{ }_{(0)}, \\
& \lambda_{2}^{n}\left(a^{1} \otimes \ldots \otimes a^{n}\right)=a^{1}{ }_{(-1)} \otimes \ldots \otimes a^{n}{ }_{(-1)} \otimes a^{1}{ }_{(0)} \ldots a^{n}{ }_{(0)},
\end{aligned}
$$

for all $a^{1} \ldots a^{n} \in A$. Define also an $A$-bimodule structure on $H^{\otimes n} \otimes_{\mathbb{k}} A$ via the maps

$$
\beta_{l}^{n}: A \otimes_{\mathbb{k}} H^{\otimes n} \otimes_{\mathbb{k}} A \rightarrow H^{\otimes n} \otimes_{\mathbb{k}} A, \quad \beta_{r}^{n}: H^{\otimes n} \otimes_{\mathbb{k}} A \otimes_{\mathbb{k}} A \rightarrow H^{\otimes n} \otimes_{\mathbb{k}} A,
$$

by

$$
\begin{aligned}
& \beta_{l}^{n}\left(a \otimes y^{1} \otimes \ldots \otimes y^{n} \otimes b\right)=\lambda_{l}^{n}\left(a_{(-1)} \otimes y^{1} \otimes \ldots \otimes y^{n}\right) \otimes a_{(0)} b, \\
& \beta_{r}^{n}\left(y^{1} \otimes \ldots \otimes y^{n} \otimes a \otimes b\right)=\lambda_{r}^{n}\left(y^{1} \otimes \ldots \otimes y^{n} \otimes b_{(-1)}\right) \otimes a b_{(0)},
\end{aligned}
$$

for all $y^{1}, \ldots, y^{n} \in H, a, b \in A$. For $n=0$ we define $\beta_{l}^{0}=\mu=\beta_{r}^{0}$. We also shall need for any $n \in \mathbb{N}$ and $1 \leq i \leq n$ the maps

$$
\mu_{i}^{n}: A^{\otimes n+1} \rightarrow A^{\otimes n+1}, \quad \mu_{i}^{n}\left(a^{1} \otimes \ldots \otimes a^{n+1}\right)=a^{1} \otimes \ldots \otimes a^{i} a^{i+1} \otimes \ldots \otimes a^{n+1},
$$

for all $a^{1}, \ldots, a^{n+1} \in A$.

Let us recall the bicomplex $C^{p, q}(A)$ defined by

$$
C^{p, q}(A)= \begin{cases}0 & \text { if } p=0 \\ \operatorname{Der}(A) & \text { if }(p, q)=(1,0) \\ \operatorname{Hom}_{\mathbb{k}}\left(A^{\otimes p}, H^{\otimes q} \otimes_{\mathbb{k}} A\right) & \text { otherwise. }\end{cases}
$$

Define the horizontal and vertical differentials

$$
\partial_{p, q}^{h}: C^{p, q}(A) \rightarrow C^{p+1, q}(A), \quad \partial_{p, q}^{v}: C^{p, q}(A) \rightarrow C^{p, q+1}(A),
$$

by

$$
\partial_{p, q}^{h}=\sum_{i=0}^{p+1}(-1)^{i} \partial_{p, q}^{h}[i], \quad \partial_{p, q}^{v}=\sum_{i=0}^{q+1}(-1)^{i} \partial_{p, q}^{v}[i] .
$$

Where for any $f \in C^{p, q}(A)$

$$
\partial_{p, q}^{h}[i](f)= \begin{cases}\beta_{l}^{q}\left(i d_{A} \otimes f\right) & \text { if } i=0 \\ f \circ \mu_{i}^{p} & \text { if } 1 \leq i \leq p \\ \beta_{r}^{q}\left(f \otimes \mathrm{id}_{A}\right) & \text { if } i=p+1\end{cases}
$$

and 


$$
\partial_{p, q}^{v}[i](f)= \begin{cases}\left(\operatorname{id}_{H} \otimes f\right) \lambda_{1}^{p} & \text { if } i=0 \\ \left(\Delta_{i}^{q} \otimes \operatorname{id}_{A}\right) f & \text { if } 1 \leq i \leq q \\ \left(\operatorname{id}_{H \otimes q} \otimes \lambda\right) f & \text { if } i=q+1 .\end{cases}
$$

We now define a new complex. For each $p, q$ set $\mathcal{C}^{p, q}(A, H)=C^{p, q}(A) \oplus$ $C^{p, q+1}(H)$. Define the horizontal and vertical differentials by

$$
d_{p, q}^{h}: \mathcal{C}^{p, q}(A, H) \rightarrow \mathcal{C}^{p+1, q}(A, H), \quad d_{p, q}^{v}: \mathcal{C}^{p, q}(A, H) \rightarrow \mathcal{C}^{p, q+1}(A, H)
$$

by the formulas

$$
d_{p, q}^{h}(\phi, f)=\left(\partial_{p, q}^{h} \phi, \partial_{p, q+1}^{h} f\right)
$$

and

$$
d_{p, q}^{v}(\phi, f)=\left(\partial_{p, q}^{v} \phi+(-1)^{q}\left(f \otimes \operatorname{id}_{A}\right) \lambda_{2}^{p}, \partial_{p, q+1}^{v} f\right) .
$$

Here we abuse of the notation by using the same symbol for denoting the differentials coming from the bialgebra cohomology of $H$ and those coming from the cohomology of the comodule algebra $A$.

Lemma 6.1. $\mathcal{C}^{*, *}(A, H)=\left(\mathcal{C}^{p, q}(A, H), d_{p, q}^{h},(-1)^{p} d_{p, q}^{v}\right)$ is a bicomplex.

Proof. We have to verify that the following equations hold: $d^{h} \circ d^{h}=0$, $d^{v} \circ d^{v}=0$ and $d^{h} \circ d^{v}=d^{v} \circ d^{h}$. The first one is obvious. Let us prove the other two.

It can be easily checked, case by case, that for any $0 \leq i \leq q+2$ and any $0 \leq j \leq p+1$ the equalities

$$
\begin{gathered}
\partial_{p, q+1}^{v}[i]\left(\left(f \otimes \mathrm{id}_{A}\right) \lambda_{2}^{p}\right)=\left(\partial_{p, q}^{v}[i](f) \otimes \mathrm{id}_{A}\right) \lambda_{2}^{p}, \\
\partial_{p, q+1}^{h}[j]\left(\left(f \otimes \mathrm{id}_{A}\right) \lambda_{2}^{p}\right)=\left(\partial_{p, q+1}^{h}[j](f) \otimes \mathrm{id}\right) \lambda_{2}^{p+1}
\end{gathered}
$$

hold. This implies that

$$
\partial_{p, q+1}^{v}\left(\left(f \otimes \mathrm{id}_{A}\right) \lambda_{2}^{p}\right)=\left(\partial_{p, q}^{v}(f) \otimes \mathrm{id}_{A}\right) \lambda_{2}^{p}
$$

and

$$
\partial_{p, q+1}^{h}\left(\left(f \otimes \mathrm{id}{ }_{A}\right) \lambda_{2}^{p}\right)=\left(\partial_{p, q+1}^{h}(f) \otimes \mathrm{id}\right) \lambda_{2}^{p+1} .
$$

Let $p, q \in \mathbb{N}$ and $(\phi, f) \in \mathcal{C}^{p, q}(A, H)$ then

$$
\begin{aligned}
d_{p, q+1}^{v} d_{p, q}^{v}(\phi, f) & =d_{p, q+1}^{v}\left(\partial_{p, q}^{v} \phi+(-1)^{q}\left(f \otimes \mathrm{id}_{A}\right) \lambda_{2}^{p}, \partial_{p, q}^{v} f\right) \\
& =\left(\partial_{p, q+1}^{v} \partial_{p, q}^{v} \phi+(-1)^{q} \partial_{p, q+1}^{v}\left(\left(f \otimes \mathrm{id}_{A}\right) \lambda_{2}^{p}\right)+\right. \\
& \left.(-1)^{q+1}\left(\partial_{p, q}^{v} f \otimes \mathrm{id}_{A}\right) \lambda_{2}^{p}, \partial_{p, q+1}^{v} \partial_{p, q}^{v} f\right) .
\end{aligned}
$$

Follows from (6.1) that this last expression is zero. By a straightforward computation one proves that $d_{p+1, q}^{v} d_{p, q}^{h}=d_{p, q+1}^{h} d_{p, q}^{v}$ using (6.2). 
The total complex $\left(\operatorname{Tot}^{*}\left(\mathcal{C}^{*, *}(A, H)\right), d\right)$ of the bicomplex $\mathcal{C}^{*, *}(A, H)$ is defined by

$$
\operatorname{Tot}^{n}\left(\mathcal{C}^{*, *}(A, H)\right)=\bigoplus_{p+q=n} C^{p, q}(A) \oplus \bigoplus_{p+q=n} C^{p, q+1}(H)
$$

and the differentials $d^{n}: \operatorname{Tot}^{n}\left(\mathcal{C}^{*, *}(A, H)\right) \rightarrow \operatorname{Tot}^{n+1}\left(\mathcal{C}^{*, *}(A, H)\right)$ are defined by

$$
\left.d^{n}\right|_{\mathcal{C}^{n-i, i}(A, H)}=d_{n-i, i}^{h}+(-1)^{p} d_{n-i, i}^{v}
$$

for each $0 \leq i \leq n$. Borrowing the notation of $\mathrm{D}$. Yau [Y08, we will denote the cohomology groups of this complex by $H_{c a}^{*}(A, H)$.

Let $(\phi, f) \in \mathcal{C}^{2,0}(A, H)=C^{2,0}(A) \oplus C^{2,1}(H),(\psi, g) \in \mathcal{C}^{1,1}(A, H)=$ $C^{1,1}(A) \oplus C^{1,2}(H)$. The element $(\phi, f)+(\psi, g) \in Z_{c a}^{2}(A, H)$ if and only if

$$
\begin{gathered}
\partial_{2,0}^{h} \phi=0, \quad \partial_{1,1}^{v} \psi-\left(g \otimes \operatorname{id}_{A}\right) \lambda=0 \\
\partial_{2,1}^{h} f=0=\partial_{1,2}^{v} g, \quad \partial_{2,1}^{v} f+\partial_{1,2}^{h} g=0, \\
\partial_{2,0}^{v} \phi+\left(f \otimes \operatorname{id}_{A}\right) \lambda_{2}^{2}+\partial_{1,1}^{h} \psi=0 .
\end{gathered}
$$

The next result follows from a simple computation.

Proposition 6.2. Let $H$ be a graded Hopf algebra, $(G, \mu, \lambda)$ a graded left $H$-comodule algebra. Let $\left(H[t] /\left(t^{l+1}\right), m^{l}, \Delta^{l}\right)$ be an l-deformation of $H$ and $\left(G[t] /\left(t^{l+1}\right), \mu^{l}, \lambda^{l}\right)$ an l-deformation of $G$ compatible with the deformation of $H$. Then $\left(\mu_{1}^{l}+m_{1}^{l}\right)+\left(\lambda_{1}^{l}, \Delta_{1}^{l}\right) \in Z_{c a}^{2}(G, H)$.

\section{Comodule algebras over pointed Hopf Algebras}

Let $H$ be a Hopf algebra and $R$ be a braided Hopf algebra in ${ }_{H}^{H} \mathcal{Y D}$.

Definition 7.1. A braided left comodule algebra over $R$ is an algebra $B \in$ ${ }_{H}^{H} \mathcal{Y} \mathcal{D}$ together with a linear map $\delta_{B}: B \rightarrow R \otimes_{\mathbb{k}} B, \delta_{B}(x)=x^{[-1]} \otimes x^{[0]}$, such that $\delta_{B}$ is a morphism in the category ${ }_{H}^{H} \mathcal{Y} \mathcal{D},\left(B, \delta_{B}\right)$ is a left $R$-comodule, and such that for all $x, y \in B$

$$
\delta_{B}(x y)=x^{[-1]}\left(x^{[0]}(-1) \cdot y^{[-1]}\right) \otimes x^{[0]}{ }_{(0)} y^{[0]} .
$$

7.1. Comodule algebras over bosonizations. We shall give a simple recipe to produce comodule algebras over a Hopf algebra constructed from a bosonization.

Let $H_{0}$ be a Hopf algebra and $R \in{ }_{H_{0}}^{H_{0}} \mathcal{Y D}$ be a braided Hopf algebra. Let us recall that the bosonization, or Radford biproduct, $H=R \# H_{0}$, is the Hopf algebra over the vector space $R \otimes_{\mathbb{k}} H_{0}$ with product and coproduct given by

$$
\begin{gathered}
(r \# g)(s \# f)=r g_{(1)} \cdot s \# g_{(2)} f, \\
\Delta(r \# g)=r_{(1)} \#\left(r_{(2)}\right)_{(-1)} g_{(1)} \otimes\left(r_{(2)}\right)_{(0)} \# g_{(2)},
\end{gathered}
$$

for all $r, s \in R, f, g \in H$. 
Let $B$ be a braided comodule algebra over $R$ and $F$ be a left $H_{0}$-comodule algebra. We shall give to the tensor product $B \otimes_{\mathbb{k}} F$ a natural structure of $R \# H_{0^{-}}$comodule algebra. Let us define the product and left $R \# H_{0^{-}}$ comodule structure by:

$$
\begin{gathered}
\delta(x \otimes f)=x^{[-1]} \# x_{(-1)}^{[0]} f_{(-1)} \otimes x^{[0]}{ }_{(0)} \otimes f_{(0)}, \\
(x \otimes f)(y \otimes g)=x\left(f_{(-1)} \cdot y\right) \otimes f_{(0)} g,
\end{gathered}
$$

for all $x, y \in B, f, g \in F$. We shall denote by $B \# F$ the space $B \otimes_{\mathbb{k}} F$ together this new product and the coaction $\delta$.

Proposition 7.2. The following assertions hold:

(1) $B \# F$ is a left $R \# H_{0}$-comodule algebra.

(2) If $F^{\mathrm{co} H_{0}}=\mathbb{k}$ and $B^{\mathrm{co} R}=\mathbb{k}$ then we have that $(B \# F)^{\mathrm{co} R \# H_{0}}=\mathbb{k}$.

Proof. (1) follows by a straightforward computation.

Let $\sum x_{i} \otimes f_{i} \in(B \# F)^{\text {co } R \# H_{0}}$. Then

$$
\delta\left(\sum x_{i} \otimes f_{i}\right)=\sum x_{i}^{[-1]} \# x_{i}^{[0]}{ }_{(-1)} f_{i(-1)} \otimes x_{i}^{[0]}{ }_{(0)} \otimes f_{i(0)}=\sum 1 \otimes 1 \otimes x_{i} \otimes f_{i} .
$$

Applying the counit on the second tensorand we obtain that

$$
\sum x_{i}^{[-1]} \otimes x_{i}^{[0]} \otimes f_{i}=\sum 1 \otimes x_{i} \otimes f_{i},
$$

whence $x_{i} \in B^{\mathrm{co} R}=\mathbb{k}$, which implies that $f_{i} \in F^{\mathrm{co} H_{0}}=\mathbb{k}$. This proves (2).

7.2. Loewy-graded comodule algebras over pointed Hopf algebras.

For the rest of this section $G$ will denote a finite group, $V \in \mathbb{k}_{\mathbb{k} G}^{\mathbb{V} G} \mathcal{\mathcal { D }}$ will denote a Yetter-Drinfeld module over $G$ and $\mathfrak{B}(V)$ the associated Nicholas algebra, see AS02 and references therein. The Nichols algebra is a $\mathbb{N}_{0^{-}}$ graded braided Hopf algebra with grading $\mathfrak{B}(V)=\oplus_{i=0}^{m} \mathfrak{B}^{i}(V)$. Let us denote by $\delta: V \rightarrow \mathbb{k}[G] \otimes_{\mathbb{k}} V$ the coaction.

We denote the bosonization $H=\mathfrak{B}(V) \# \mathbb{k}[G]$. The Hopf algebra $H$ is a coradically graded Hopf algebra, with grading given by $H(n)=\mathfrak{B}^{n}(V) \otimes_{\mathbb{k}} \mathbb{k}[G]$ for any $n=0 \ldots m$. We will denote by $\theta: H \rightarrow \mathfrak{B}(V)$ and $p: H \rightarrow \mathbb{k}[G]$ the canonical projections. For any $h \in H$ we have that $h=\theta\left(h_{(1)}\right) p\left(h_{(2)}\right)$. The space $\mathfrak{B}(V)$ are the coinvariants of $H$ under the coaction (id $\otimes p$ ) $\Delta$, that is $\mathfrak{B}(V)=\{h \in h:($ id $\otimes p) \Delta(h)=h \otimes 1\}$.

Let $(A, \lambda)$ be a Loewy-graded left $H$-comodule algebra with gradation given by $A=\bigoplus_{i=0}^{m} A(i)$. Let $\pi: A \rightarrow A(0)$ be the canonical projection.

Let us define

$$
\mathfrak{B}_{A}=\{a \in A:(\operatorname{id} \otimes \pi) \lambda(a) \in H \otimes 1\} .
$$

This space should be thought as a kind of diagram [AS02, for the comodule algebra $A$. Indeed, if $(A, \lambda)=(H, \Delta)$ then $\mathfrak{B}_{A}=\mathfrak{B}(V)$ is the diagram corresponding to $H$. 
For each $n \in \mathbb{N}$ define $\mathfrak{B}_{A}(n)=\mathfrak{B}_{A} \bigcap A(n)$.

Proposition 7.3. The following statements holds.

(1) $\mathfrak{B}_{A}(0)=\mathbb{k} 1, \mathfrak{B}_{A}=\bigoplus_{n=0} \mathfrak{B}_{A}(n)$.

(2) $\mathfrak{B}_{A} \subseteq A$ is a left $H$-subcomodule subalgebra, in particular $\lambda\left(\mathfrak{B}_{A}(n)\right) \subseteq$ $\oplus_{i=0}^{n} H(n-i) \otimes_{\mathbb{k}} \mathfrak{B}_{A}(i)$.

(3) There exists an injective $H$-comodule algebra homomorphism $\iota$ : $\mathfrak{B}_{A} \rightarrow \mathfrak{B}(V)$ such that $\iota\left(\mathfrak{B}_{A}(n)\right) \subseteq \mathfrak{B}^{n}(V)$ for any $n=0 \ldots m$.

(4) If $\mathfrak{B}_{A}(1)=0$ then $\mathfrak{B}_{A}=\mathbb{k} 1$.

(5) The space $\iota\left(\mathfrak{B}_{A}(1)\right) \subseteq V$ is a $\mathbb{k} G$-subcomodule.

(6) The multiplication map $\mu: \mathfrak{B}_{A} \otimes_{\mathbb{k}} A_{0} \rightarrow A$ is injective.

Proof. (1). The first assertion is clear. Let $a \in \mathfrak{B}_{A}$, then $a=\sum_{i=0} a_{i}$, where $a_{i} \in A(i)$. Since $(\mathrm{id} \otimes \pi) \lambda(a) \in H \otimes_{\mathbb{k}} 1$ and the spaces $H(i)$ are disjoint we conclude that $(\mathrm{id} \otimes \pi) \lambda\left(a_{i}\right) \in H(i) \otimes_{\mathbb{k}} 1$ for each $i$, hence $a_{i} \in \mathfrak{B}_{A}(i)$.

(2). It is clear that $\mathfrak{B}_{A}$ is a subalgebra since $\lambda$ and $\pi$ are algebra maps. Let $a \in \mathfrak{B}_{A}$, then $a_{(-1)} \otimes \pi\left(a_{(0)}\right)=h \otimes 1$, for some $h \in H$. Then

$$
\Delta(h) \otimes 1=a_{(-2)} \otimes a_{(-1)} \otimes \pi\left(a_{(0)}\right)=a_{(-1)} \otimes a_{(0)(-1)} \otimes \pi\left(a_{(0)(0)}\right) .
$$

Therefore $a_{(-1)} \otimes a_{(0)} \in H \otimes \mathfrak{B}_{A}$.

(3). For $a \in \mathfrak{B}_{A}$ define the map $\iota: \mathfrak{B}_{A} \rightarrow H$ by $\iota(a)=h \in H$, where $h$ is the unique element in $H$ such that $a_{(-1)} \otimes \pi\left(a_{(0)}\right)=h \otimes 1$. Using (7.2) it is easy to see that $\iota$ is an $H$-comodule map.

Let us prove that $\iota$ is injective. Let $a \in \mathfrak{B}_{A}$ such that $\iota(a)=0$, thus $a_{(-1)} \otimes \pi\left(a_{(0)}\right)=0$. Let us write $a=\sum_{n} a_{n}$ where $a_{n} \in A(n)$. In this case $\lambda(a)=\sum_{n} \lambda\left(a_{n}\right)=\sum_{n} \sum_{i=0}^{n} b_{i}^{n}$, where $b_{i}^{n} \in H(i) \otimes_{\mathbb{k}} A(n-i)$, for $i=0 \ldots n$. Since $a_{(-1)} \otimes \pi\left(a_{(0)}\right)=\sum_{n} b_{n}^{n}=0$ this means that for each $n, b_{n}^{n}=0$, thus $\lambda\left(a_{n}\right) \in H_{n-1} \otimes_{\mathbb{k}} A$, whence $a_{n} \in A_{n-1}$, which is impossible unless $a_{n}=0$ since for all $n$ : $A(n) \bigcap A_{n-1}=0$. Thus $\iota$ is injective.

Observe that $\lambda \pi=(p \otimes \pi) \lambda$, thus if $a \in \mathfrak{B}_{A}$ then there exists an element $h \in H$ such that $a_{(-1)} \otimes \pi\left(a_{(0)}\right)=h \otimes 1$. Hence

$$
\begin{aligned}
(\mathrm{id} \otimes p) \Delta)(h) \otimes 1 & =a_{(-2)} \otimes p\left(a_{(-1)}\right) \otimes \pi\left(a_{(0)}\right)=a_{(-1)} \otimes(p \otimes \pi) \lambda\left(a_{(0)}\right) \\
& =a_{(-1)} \otimes \lambda \pi\left(a_{(0)}\right)=h \otimes 1 \otimes 1 .
\end{aligned}
$$

Thus (id $\otimes p) \Delta(h)=h \otimes 1$, that is $h \in \mathfrak{B}(V)$. Since $H(n)=\mathfrak{B}^{n}(V) \# \mathbb{k} G$ and $\iota$ is an $H$-comodule map then $\iota\left(\mathfrak{B}_{A}(n)\right) \subseteq \mathfrak{B}^{n}(V)$. This ends the proof of (3).

(4). Let us assume that $\mathfrak{B}_{A}(1)=0$. We can (and will) assume that $\mathfrak{B}_{A} \subseteq \mathfrak{B}(V)$. Let $a \in \mathfrak{B}_{A}(2)$ then $\Delta(a) \in H_{0} \otimes_{\mathbb{k}} \mathfrak{B}_{A}(2) \oplus H(2) \otimes_{\mathbb{k}} \mathfrak{B}_{A}(0)$, thus $a \in H_{1}$, hence $a=0$. Using the same argument we can prove inductively that $\mathfrak{B}_{A}(n)=0$ for all $n \geq 2$.

(5). Let us consider $W=\mathfrak{B}_{A}(1)$ as a $\mathbb{k} G$-comodule via $\left(p \otimes \mathrm{id}_{A}\right) \lambda$. Then $W=\oplus_{g \in G} W_{g}$, where $W_{g}=\left\{w \in \mathfrak{B}_{A}(1): p\left(a_{(-1)}\right) \otimes a_{0}=g \otimes a\right\}$. If $a \in W_{g}$ 
then $\lambda(a)=g \otimes a+v_{a} \otimes 1$, for some $v_{a} \in R(1)$. Note that $\iota(a)=v_{a}$. In this case, $\delta\left(v_{a}\right)=g \otimes v_{a}$, thus $\iota(W)$ is a subcomodule of $V$.

Now, let us prove (6). Consider the map $\phi: A \rightarrow \mathfrak{B}(V) \otimes_{\mathbb{k}} A_{0}$ given by $\phi(a)=\theta\left(a_{(-1)}\right) \otimes \pi\left(a_{(0)}\right)$ and let $\mu: \mathfrak{B}_{A} \otimes_{\mathbb{k}} A_{0} \rightarrow A$ be the multiplication map. Then $\phi \circ \mu=\iota \otimes \mathrm{id}_{A_{0}}$, indeed if $x \in \mathfrak{B}_{A}, a \in A_{0}$ then

$$
\begin{aligned}
\phi(x a) & =\theta\left(x_{(-1)} a_{(-1)}\right) \otimes \pi\left(x_{(-1)} a_{(0)}\right)=\theta\left(x_{(-1)} a_{(-1)}\right) \otimes \pi\left(x_{(-1)}\right) a_{(0)} \\
& =\theta\left(\iota(x) a_{(-1)}\right) \otimes a_{(0)}=\iota(x) \otimes a .
\end{aligned}
$$

Since $\iota$ is injective this implies that $\mu$ is injective.

Remark 7.4. The space $\iota\left(\mathfrak{B}_{A}\right)$ is a left homogeneous coideal subalgebra of $R$. Even if $R$ is a Nichols algebra, $\mathfrak{B}_{A}$ need not be generated by $\mathfrak{B}_{A}(1)$. However, in some special cases, for example when $R$ is a quantum linear space, $\mathfrak{B}_{A}$ is always generated in degree one.

Let us further assume that $(A, \lambda)$ is right $H$-simple. Follows by Proposition 4.4 that $A(0)$ is right $\mathbb{k}[G]$-simple and therefore there exists a subgroup $F \subseteq G$ and a 2-cocycle $\psi \in Z^{2}\left(F, \mathbb{k}^{\times}\right)$such that $A(0) \simeq \mathbb{k}^{\psi}[F]$ as $\mathbb{k}[G]$ comodule algebras. That is, there is a basis $\left\{e_{f}\right\}_{f \in F}$ of $A(0)$ such that

$$
e_{f} e_{f^{\prime}}=\psi\left(f, f^{\prime}\right) e_{f f^{\prime}}, \quad \lambda\left(e_{f}\right)=f \otimes e_{f},
$$

for all $f, f^{\prime} \in F$.

In the case when $\psi$ is trivial there is an action of $F$ on $\mathfrak{B}_{A}$ given as follows. If $x \in \mathfrak{B}_{A}$ and $f \in F$ then

$$
f \cdot x:=e_{f} x e_{f^{-1}} \in \mathfrak{B}_{A} .
$$

The coaction $\delta: \mathfrak{B}_{A} \rightarrow \mathbb{k}[F] \otimes_{\mathbb{k}} \mathfrak{B}_{A}$, given by $\delta(x)=p\left(x_{(-1)}\right) \otimes x_{(0)}$ makes $\mathfrak{B}_{A}$ into a Yetter-Drinfeld module over $\mathbb{k}[F]$. Thus, we can consider the smash product $\mathfrak{B}_{A} \# A(0)$ as in Proposition 7.2 .

The following proposition tells us that the comodule algebra $A$ can be recovered from $\mathfrak{B}_{A}$ and $A_{0}$.

Proposition 7.5. Assume that the group $G$ is a cyclic group, then $A \simeq$ $\mathfrak{B}_{A} \# A(0)$ as $H$-comodule algebras.

Proof. Since $G$ is cyclic then $\psi$ must be trivial and $A_{0}$ is isomorphic to the group algebra $\mathbb{k} F$, where $F \subseteq G$ is a subgroup. Thus, we can consider the product $\mathfrak{B}_{A} \# A(0)$.

By Proposition 7.3 (6) the multiplication map $\mu: \mathfrak{B}_{A} \# A_{0} \rightarrow A$ is an injective $H$-comodule algebra map. Let us prove that $\mu$ is bijective.

Let $Q$ denote the quotient coalgebra $H /(\mathbb{k} F)^{+} H$ and $\gamma: H \rightarrow Q$ the canonical projection. Since $A \in{ }_{\mathbb{k} F}^{H} \mathcal{M}$, then $A \simeq H \square_{Q} \bar{A}$, where $\bar{A}=$ $A /(\mathbb{k} F)^{+} A$ and the left $Q$-comodule structure on $\bar{A}$ is given by $\left(\gamma \otimes \mathrm{id}_{A}\right) \lambda$. The left $H$-comodule structure on $H \square_{Q} \bar{A}$ is given by the coproduct of $H$. 
There is an isomorphism $\alpha: \mathbb{k} F \otimes Q \stackrel{\simeq}{\longrightarrow} H$ of left $\mathbb{k} F$-modules, right $Q$-comodules. Thus there is a linear isomorphism $A \simeq \mathbb{k} F \otimes \bar{A}$.

It is easy to see that $\left(H \square_{Q} \bar{A}\right)_{0}=\mathbb{k} F \otimes \overline{1}$. Let us denote by $\pi: H \square_{Q} \bar{A} \rightarrow$ $\left(H \square_{Q} \bar{A}\right)_{0}$ the projection. The image of the injective map $\beta: \bar{A} \rightarrow H \square_{Q} \bar{A}$ given by $\beta(\bar{a})=\alpha\left(1 \otimes \bar{a}_{(-1)}\right) \otimes \bar{a}_{(0)}$ composed with the isomorphism $A \simeq$ $H \square_{Q} \bar{A}$ is inside $\mathfrak{B}_{A}$. Indeed, for any $\bar{a} \in \bar{A}$ the element

$$
\alpha\left(1 \otimes \bar{a}_{(-1)}\right)_{(1)} \otimes \pi\left(\alpha\left(1 \otimes \bar{a}_{(-1)}\right)_{(2)} \otimes \bar{a}_{(0)}\right) \in H \otimes 1 .
$$

This implies that $\operatorname{dim} \bar{A} \leq \operatorname{dim} \mathfrak{B}_{A}$. Whence, $\operatorname{dim} A \leq \mathfrak{B}_{A}|F|$, thus $\mu$ is surjective and therefore a bijection.

Remark 7.6. Assume we are under the hypothesis of Proposition 7.5, It is immediate to verify that under the identification of $A(0) \simeq \mathbb{k} F \hookrightarrow \mathbb{k} G$, the space $\iota\left(\mathfrak{B}_{A}\right) \subseteq \mathfrak{B}(V)$ is stable under the action of $F$.

\section{Some Classification Results}

Let $H$ be a pointed Hopf algebra with coradical the group algebra of a cyclic group $C_{n}$, such that $\operatorname{gr}_{c} H=\mathfrak{B}(V) \# \mathbb{k}\left[C_{n}\right]$, where $V \in{ }_{\mathbb{k} C_{n}}^{\mathbb{k} C_{n}} \mathcal{Y} D$ is a Yetter-Drinfeld module and $\mathfrak{B}(V)$ the associated Nichols algebra.

We propose the following strategy to classify module categories over the category $\operatorname{Rep}(H)$. First we determine all Loewy-graded right $\mathfrak{B}(V) \# \mathbb{k}[G]$ simple left $\mathfrak{B}(V) \# \mathbb{k}[G]$-comodule algebras $A$, with trivial coinvariants. By Proposition 7.5 this comodule algebras are determined by the subalgebra $\mathfrak{B}_{A}$ and $A_{0}$, where $\mathfrak{B}_{A}=\oplus_{i} \mathfrak{B}_{A}(i)$ is a homogeneous left coideal subalgebra of $\mathfrak{B}(V)$ stable under the action of $A_{0}$. Since $A_{0}$ is a $\mathbb{k} C_{n}$-simple left $\mathbb{k} C_{n^{-}}$ comodule algebra, then $A_{0}=\mathbb{k} C_{d}$ for some divisor $d$ of $n$. In our examples $\mathfrak{B}_{A}$ is generated as an algebra by $\mathfrak{B}_{A}(1)$, so really $\mathfrak{B}_{A}$ depends on a subspace $W \subseteq V$ stable under the action of $C_{d}$. To end we shall find all possible liftings of this comodule algebras.

In this section we present the classification of exact module categories over some Hopf algebras, where the dimension of $V$ is 1 or 2 . More precisely we will show the classification for the Taft Hopf algebras $T_{q}$, over the Radford Hopf algebras $\mathbf{r}_{q}$, over the book Hopf algebras and over the small quantum groups $u_{q}\left(\mathfrak{s l}_{2}\right)$.

Let us recall the definition of these Hopf algebras and some other Hopf algebras that will be used later. Some notations are taken from AS98. Let $n \in \mathbb{N}$ and $q$ be a $n$-th primitive root of unity.

- The Taft Hopf algebra $T_{q}=\mathbb{k}\left\langle g, x \mid g x=q x g, g^{n}=1, x^{n}=0\right\rangle$, with coproduct determined by $\Delta(g)=g \otimes g, \Delta(x)=x \otimes 1+g \otimes x$.

- The algebra $\widehat{T(q)}=\mathbb{k}\left\langle g, x \mid g x=q x g, g^{n^{2}}=1, x^{n}=0\right\rangle$. The coproduct is determined by $\Delta(x)=x \otimes 1+g \otimes x, \Delta(g)=g \otimes g$. 
- The Radford Hopf algebra is $\mathbf{r}(q)=\mathbb{k}\langle g, x| g x=q x g, g^{n^{2}}=1, x^{n}=$ $\left.1-g^{n}\right\rangle$. The coproduct is determined by $\Delta(g)=g \otimes g, \Delta(x)=$ $g \otimes x+x \otimes 1$.

- The book Hopf algebras $\mathcal{H}(1, q)=\mathbb{k}\langle g, x, y| g^{n}=1, g x=q x g$, gy $=$ $\left.q^{-1} y g, x y=q y x, x^{n}=0=y^{n}\right\rangle$. With coproduct determined by $\Delta(g)=g \otimes g, \Delta(x)=x \otimes 1+g^{-1} \otimes x, \Delta(y)=y \otimes 1+g^{-1} \otimes y$.

- The Frobenius-Lusztig kernel $u_{q}\left(\mathfrak{s l}_{2}\right)=\mathbb{k}\langle g, x, y| g x=q^{2} x g$, gy= $\left.q^{-2} y g, g^{n}=1, x^{n}=0=y^{n}, x y-y x=g-g^{-1}\right\rangle$. The coproduct is determined by $\Delta(g)=g \otimes g, \Delta(x)=x \otimes g+1 \otimes x, \Delta(y)=$ $y \otimes 1+g^{-1} \otimes y$.

8.1. Module categories over $\operatorname{Rep}\left(T_{q}\right)$. In this section we recover, using different techniques, the results obtained in [EO04, Thm 4.10].

Let $d$ be a divisor of $n$. Set $n=d m$. For any $\xi \in \mathbb{k}$ define the algebra $\mathcal{A}(d, \xi)$ generated by elements $h, w$ subject to relations

$$
h^{d}=1, \quad h w=q^{m} w h, \quad y^{n}=\xi 1 .
$$

The algebra $\mathcal{A}(d, \xi)$ is a left $T_{q}$-comodule algebra with coaction determined by

$$
\lambda(h)=g^{m} \otimes h, \lambda(w)=x \otimes 1+g \otimes w .
$$

Let us denote by $K(d)$ the left coideal subalgebra of $T_{q}$ generated by $g^{m}$ and $x$.

Lemma 8.1. For any $\xi \in \mathbb{k}$ there is an isomorphism $\mathcal{A}(d, \xi) \simeq K(d)_{\sigma_{\xi}}$ for some cocentral 2-cocycle $\sigma_{\xi}$. In particular the algebras $\mathcal{A}(d, \xi)$ are right $T_{q}$-simple left $T_{q}$-comodule algebras such that $\mathcal{A}(d, \xi)^{\text {co } T_{q}}=\mathbb{k}$.

Proof. The algebra $\mathcal{A}(n, \xi)$ is a left $T_{q}$-Galois extension and $\mathcal{A}(d, \xi) \subset \mathcal{A}(n, \xi)$ is a subalgebra and a left $T_{q^{-}}$-submodule. Thus there exists a 2-cocycle such that $\mathcal{A}(n, \xi) \simeq \sigma_{\xi} T_{q}$. This cocycle is cocentral since $\mathcal{A}(n, \xi)$ is also a right $T_{q}$-comodule algebra with structure map $\rho: \mathcal{A}(n, \xi) \rightarrow \mathcal{A}(n, \xi) \otimes_{\mathbb{k}} T_{q}$ given by

$$
\rho(h)=h \otimes g, \quad \rho(w)=w \otimes 1+h \otimes x .
$$

The other statements are straightforward.

Lemma 8.2. (i) Categories ${ }_{\mathcal{A}(d, \xi)} \mathcal{M}$ are semisimple for any divisor $d$ of $n$ and any $\xi \in \mathbb{k}^{\times}$.

(ii) For any $\xi \in \mathbb{k}^{\times}$the rank of $\mathcal{A}(d, \xi) \mathcal{M}$ is $\frac{n}{d}$.

(iii) The algebras $\mathcal{A}(d, \xi), \mathcal{A}\left(d^{\prime}, \xi^{\prime}\right)$ are Morita equivariant equivalent if and only if $d=d^{\prime}, \xi=\xi^{\prime}$.

Proof. (i) and (ii) are Straightforward. Let us assume that $\mathcal{A}(d, \xi) \sim_{M}$ $\mathcal{A}\left(d, \xi^{\prime}\right)$. From (ii) we get that $d=d^{\prime}$. Let us denote by $Q$ the quotient coalgebra $Q=T_{q} / K(d)^{+} T_{q} \simeq \mathbb{k} C_{m}$. Using Lemma 3.6 and Lemma 8.1 there exists a group-like element $f \in G\left(T_{q}\right)$ such that $\mathcal{A}\left(d, \xi^{\prime}\right) \simeq\left(f K(d) f^{-1}\right)_{\sigma_{\xi}}$. Thus $\mathcal{A}\left(d, \xi^{\prime}\right) \simeq \mathcal{A}(d, \xi)$, whence $\xi=\xi^{\prime}$. 
Proposition 8.3. If $\mathcal{M}$ is an exact indecomposable module category over $\operatorname{Rep}\left(T_{q}\right)$ then $\mathcal{M} \simeq{ }_{\mathbb{k} C_{d}} \mathcal{M}$ or $\mathcal{M} \simeq{ }_{\mathcal{A}(d, \xi)} \mathcal{M}$ for some divisor $d$ of $n$ and $\xi \in \mathbb{k}$.

Proof. Let $G$ be a Loewy-graded left $T_{q}$-comodule algebra. Let $d$ be a divisor of $n$ such that $G_{0}=\mathbb{k} C_{d}$. Let us assume that $G \neq G_{0}$. Using Proposition $7.5 G \simeq \mathfrak{B}_{G} \# \mathbb{k} C_{d}$. Since $G \neq G_{0}$ then $\mathfrak{B}_{G}(1) \neq 0$. The only possibility is that $G=\mathcal{A}(d, 0)$.

Let $(A, \lambda)$ be $T_{q}$-simple left $T_{q}$-comodule algebra such that $A$ is a lifting of $\mathcal{A}(d, 0)$ along $T_{q}$ for some $d$ is a divisor of $n$. So in this case $A_{0}=\mathbb{k} C_{d}$ is the group algebra of the cyclic group of $d$ elements generated by $h$ where $\lambda\left(h^{i}\right)=g^{m i} \otimes h^{i}$, for any $i=0 \ldots d-1$.

Let $y \in \mathcal{A}_{1}-\mathcal{A}_{0}$. Since $A$ is a lifting for $\mathcal{A}(d, 0)$, the set $\left\{h^{i} y^{j}: i=\right.$ $0 \ldots d-1, j=0 \ldots n-1\}$ is a basis for $A$. Here $A_{0}=\mathbb{k}\left\{h^{i}: i=0 \ldots d-1\right\}$ where $d m=n$. By Lemma 5.5 we have that $\lambda(y)=x \otimes 1+g \otimes y$.

It is easy to see that $\lambda\left(h y-q^{m} y h\right)=g^{m+1} \otimes h y-q^{m} y h$. Thus $h y-q^{m} y h \in$ $\mathcal{A}_{0}$. Therefore there are scalars $\zeta_{i} \in \mathbb{k}, i=0 \ldots d-1$ such that $h y-q^{m} y h=$ $\sum_{i} \zeta_{i} h^{i}$. Since $\lambda\left(h y-q^{m} y h\right)=g^{m+1} \otimes h y-q^{m} y h$ and $\lambda(h)=g^{m} \otimes h$ then $\zeta_{i}=0$ for all $i$, and $h y=q^{m} y h$.

Since $\lambda\left(y^{n}\right)=x^{n} \otimes 1+g^{n} \otimes y^{n}=1 \otimes y^{n}$ then $y^{n} \in A_{0}$ and there exists an $\xi \in \mathbb{k}$ such that $y^{n}=\xi 1$ and we have a projection $\mathcal{A}(d, \xi) \rightarrow A$ which is an isomorphism because both algebras have the same dimension.

8.2. Module categories over $\operatorname{Rep}\left(\mathbf{r}_{q}\right)$. Let $C_{n^{2}}$ be the cyclic group of $n^{2}$ elements generated by $g$. Let $V \in{ }_{\mathbb{k} C_{n^{2}}}^{\mathbb{k} C_{n^{2}}} \mathcal{Y \mathcal { D }}$ be the one-dimensional module generated by $w$ with action and coaction determined by

$$
g \cdot w=q w, \quad \delta(w)=g \otimes w .
$$

The following result is straightforward.

Lemma 8.4. $\operatorname{gr}_{c} \mathbf{r}(q)=\widehat{T(q)}$ and $\widehat{T(q)} \simeq \mathfrak{B}(V) \# \mathbb{k} C_{n^{2}}$.

We shall classify exact module categories over the Radford Hopf algebras in a similar way as for the Taft Hopf algebras. No new difficulty arise since the dimension of the the vector space $V$ is 1 .

Let us define families of left $\mathbf{r}(q)$-module algebras, right $\mathbf{r}(q)$-simple. Let $d$ be a divisor of $n^{2}$ and set $n^{2}=d m$. Let also $\xi \in \mathbb{k}^{\times}$.

(a) The group algebra $\mathbb{k} C_{d}=\mathbb{k}\left\langle h: h^{d}=1\right\rangle$, with coaction determined by $\lambda(h)=g^{m} \otimes h$.

(b) Algebras $\mathcal{A}(d)=\mathbb{k}\left\langle h, w: h^{d}=1, w^{n}=1, h w=q^{m} w h\right\rangle$ and coaction determined by $\lambda(h)=g^{m} \otimes h, \lambda(w)=x \otimes 1+g \otimes w$.

(c) If there is an integer $a$ such that $n a=d$, then $\mathcal{B}(a, \xi)=\mathbb{k}\langle h, w$ : $\left.h^{d}=1, w^{n}=1+\xi h^{a}, h w=q^{m} w h\right\rangle$ and coaction determined by $\lambda(h)=g^{m} \otimes h, \lambda(w)=x \otimes 1+g \otimes w$. 
For any $d$ divisor of $n^{2}$ and any $\xi \in \mathbb{k}$ the algebras $\mathcal{A}(d)$ and $\mathcal{B}(a, \xi)$ are left $\mathbf{r}(q)$-module algebras, right $\mathbf{r}(q)$-simple with trivial coinvariants. The algebras listed above are non-isomorphic as comodule algebras.

Lemma 8.5. The algebras $\mathcal{A}(d)$ and $\mathcal{B}(a, \xi)$ are twistings of left coideal subalgebras of $\boldsymbol{r}(q)$ by a compatible Hopf 2-cocycle, pair-wise non Morita equivariant equivalent.

Proof. Algebras $\mathcal{A}(n)$ and $\mathcal{B}(n, \xi)$ are $\mathbf{r}(q)$-Galois extensions of the field and $\mathcal{A}(d) \subseteq \mathcal{A}(n), \mathcal{B}(a, \xi) \subseteq \mathcal{B}(n, \xi)$ are $\mathbf{r}(q)$-subcomodules subalgebras. Let us prove now that these algebras are non Morita equivariant equivalent. For this we will need the following result:

Claim 8.1. Let $H$ be a pointed Hopf algebra. Let $J \subseteq H$ be a left coideal subalgebra and $\sigma: H \otimes_{\mathbb{k}} H \rightarrow \mathbb{k}$ a compatible 2-cocycle, such that the graded algebra $\operatorname{gr}_{l}\left({ }_{\sigma} J\right)$ is isomorphic to a left coideal subalgebra $K$ of $\operatorname{gr}_{c} H$ and the coalgebra quotient $Q=\operatorname{gr}_{c} H /\left(\operatorname{gr}_{c} H\right) K^{+}$is pointed cosemisimple. Then if $P \in{ }^{H} \mathcal{M}_{\sigma} J$ is an indecomposable object there exists a group-like element $g \in H_{0}$ such that $P \simeq g \cdot{ }_{\sigma} J$.

Idea of the proof. Consider the filtration $P_{i}=\delta^{-1}\left(H_{i} \otimes_{\mathbb{k}} P\right)$. The associated graded vector space gr $P$ is an object in the category $\operatorname{gr}_{c} H \mathcal{M}_{K} \simeq{ }^{Q} \mathcal{M}$. Since $P$ is indecomposable, the so is gr $P$. Therefore there exists a grouplike element $g \in H$ such that gr $P=g K$.

Let $A$ be a left $\mathbf{r}(q)$-comodule algebra. Let us assume, for instance, that $A \sim_{M} \mathcal{B}(a, \xi)$ for some $a$ such that $n a=d$. Then there exists an object $P \in{ }^{r(q)} \mathcal{M}_{\mathcal{B}(a, \xi)}$ such that $A \simeq \operatorname{End}_{\mathcal{B}(a, \xi)}(P)$. Since in our case we are under the hypothesis of Claim 8.1, $P=g \cdot \mathcal{B}(a, \xi)$, and arguing as in Lemma 3.6 we obtain that $A \simeq \mathcal{B}(a, \xi)$, thus if $A$ is an algebra in the list it must be equal to $\mathcal{B}(a, \xi)$. The same argument can be used to prove that if $A \sim_{M} \mathcal{A}(d)$ then $A \simeq \mathcal{A}(d)$.

Proposition 8.6. If $\mathcal{M}$ is an exact indecomposable module category over $\operatorname{Rep}(\boldsymbol{r}(q))$ then $\mathcal{M} \simeq{ }_{\mathbb{k} C_{m}} \mathcal{M}, \mathcal{M} \simeq{ }_{\mathcal{A}(d)} \mathcal{M}$ or $\mathcal{M} \simeq{ }_{\mathcal{B}(a, \xi)} \mathcal{M}$.

Proof. Let $\left(G, \lambda_{0}\right)$ be a Loewy-graded $\widehat{T(q)}$-comodule algebra. We assume that $G \neq G_{0}$ and $G_{0}=\mathbb{k}\left\langle h: h^{d}=1\right\rangle$ for some $d$ divisor of $n^{2}$. Since $\widehat{T(q)} \simeq \mathfrak{B}(V) \# \mathbb{k} C_{n^{2}}, V$ is one-dimensional and $\mathfrak{B}_{G} \subseteq \mathfrak{B}(V)$ is generated by $\mathfrak{B}_{G}(1)$, thus $G$ is generated by $h$ and an element $w$ subject to relations

$$
h^{d}=1, \quad h w=q^{m} w h, \quad w^{n}=0,
$$

Where $d m=n^{2}$. The coaction is determined by

$$
\lambda_{0}(h)=g^{m} \otimes h, \quad \lambda_{0}(w)=x \otimes 1+g \otimes w .
$$

Let $(A, \lambda)$ be a lifting of $G$ along $\mathbf{r}(q)$. In particular $A_{0}=G_{0}$. Using again Lemma 5.5 it is easy to see that $A$ is generated by elements $h$ and $w$ with $\lambda(h)=g^{m} \otimes h$, and $\lambda(w)=x \otimes 1+g \otimes w$. We can show, in a similar way as done in the proof of Proposition 8.3 , that the relation $h w=q^{m} w h$ holds. 
Since $\lambda\left(w^{n}\right)=\left(1-g^{n}\right) \otimes 1+g^{n} \otimes w^{n}$, whence $w^{n} \in A_{0}$. Thus, there exists $\xi_{i} \in \mathbb{k}, i=0 \ldots d-1$, such that $w^{n}=\sum_{i=0}^{d-1} \xi_{i} h^{i}$. Therefore

$$
\sum_{i=0}^{d-1} \xi_{i} g^{n} \otimes h^{i}+\left(1-g^{n}\right) \otimes 1=\sum_{i=0}^{d-1} \xi_{i} g^{m i} \otimes h^{i} .
$$

This implies that $\xi_{0}=1$. If $m$ does not divides $n$ then $\xi_{i}=0$ for all $i=1 \ldots d-1$, if there is an integer $a$ such that $m a=n$ then $\xi_{i}=0$ for all $i \neq a$ and $\xi_{a}$ is arbitrary. In the first case $A \simeq \mathcal{A}(d)$ and in the second case $A \simeq \mathcal{B}\left(a, \xi_{a}\right)$.

8.3. Module categories over $\operatorname{Rep}(\mathcal{H}(1, q))$. We shall assume that $n>2$. Let $V=\mathbb{k}\{x, y\}$ denote the 2-dimensional Yetter-Drinfeld module over $\mathbb{k} C_{n}$, where $C_{n}$ is the cyclic group generated by $g$, with action $\cdot: \mathbb{k} C_{n} \otimes_{\mathbb{k}} V \rightarrow V$ and coaction $\delta: V \rightarrow \mathbb{k} C_{n} \otimes_{\mathbb{k}} V$ determined by

$$
g \cdot x=q x, \quad g \cdot y=q^{-1} y, \quad \delta(x)=g^{-1} \otimes x, \quad \delta(y)=g^{-1} \otimes y .
$$

It is not difficult to prove that $\mathcal{H}(1, q)=\mathfrak{B}(V) \# \mathbb{k} C_{n}$. So $\mathcal{H}(1, q)$ is a coradically graded Hopf algebra with gradation given by $\mathcal{H}(1, q)(i)=\mathfrak{B}^{i}(V) \otimes_{\mathbb{R}} \mathbb{k} C_{n}$.

Lemma 8.7. Let $K=\oplus_{i=0}^{n-1} K(i) \subseteq \mathfrak{B}(V)$ be an homogeneous left coideal subalgebra of $\mathcal{H}(1, q)$, that is $K$ is graded as an algebra, $K(j) \subseteq \mathfrak{B}^{j}(V)$ and $\Delta(K(j)) \subseteq \oplus_{i=0}^{j} \mathcal{H}(1, q)(i) \otimes_{\mathbb{k}} K(j-i)$ for all $j=0 \ldots n-1$. Then $K$ is generated as an algebra by $K(1)$.

Proof. If $\operatorname{dim} K(1)=2$ then $K=\mathfrak{B}(V)$ and the claim is obviously true. Let us assume that $\operatorname{dim} K(1)=1$, and let $w=a x+b y$ be a nonzero element of $K(1)$. We will prove that for any $m=1 \ldots n-1, K(m)$ is the 1-dimensional vector space generated by $w^{m}$.

Let $\theta \in K(m)$, then since $K(m) \subseteq \mathfrak{B}^{m}(V), \theta=\sum_{i=0}^{m} \alpha_{i} y^{i} x^{m-i}$ for some $\alpha_{i} \in \mathbb{k}$.

Let us denote by $\chi_{i}^{n}$ the quantum Gaussian coefficients (or q-binomial coefficients), that is

$$
\chi_{i}^{n}=\frac{\left(q^{n-i+1}-1\right) \ldots\left(q^{n}-1\right)}{(q-1) \ldots\left(q^{i}-1\right)}
$$

It is well-known that

$$
\Delta\left(x^{k}\right)=\sum_{j=0}^{k} \chi_{j}^{k} g^{-j} x^{k-j} \otimes x^{j}, \quad \Delta\left(y^{k}\right)=\sum_{j=0}^{k} \chi_{j}^{k} y^{k-j} g^{-j} \otimes y^{j} .
$$

This implies that

$$
\Delta(\theta)=\sum_{i=0}^{m} \sum_{j, l} \alpha_{i} \chi_{j}^{k-i} \chi_{l}^{i}\left(y^{i-l} g^{-j-l} x^{k-i-j} \otimes y^{l} x^{j}\right) .
$$


Since $\Delta(\theta) \in \oplus_{i=0}^{m} \mathcal{H}(1, q)(m-i) \otimes K(i)$, there exists an element $v \in$ $\mathcal{H}(1, q)(m-1)$ such that the summand of $\Delta(\theta)$ that belongs to $\mathcal{H}(1, q)(m-$ $1) \otimes K(1)$ equals $v \otimes w$. Using equation (8.1) we obtain that

$$
\begin{aligned}
& a v=\sum_{i=0}^{m-1} \alpha_{i} \chi_{1}^{m-i} \chi_{0}^{i} y^{i} g^{-1} x^{m-i-1}, \\
& b v=\sum_{i=1}^{m} \alpha_{i} \chi_{0}^{m-i} \chi_{1}^{i} y^{i-1} g^{-1} x^{m-i} .
\end{aligned}
$$

When $a=0$, or $b=0$, the above equations immediately imply that $\theta$ is a scalar multiple of $y^{m}$, or $x^{m}$ respectively. Let us assume that $a b \neq 0$. In this case we have that

$$
\frac{b}{a} \sum_{i=0}^{m-1} \alpha_{i} \chi_{1}^{m-i} \chi_{0}^{i} y^{i} g^{-1} x^{m-i-1}=\sum_{i=1}^{m} \alpha_{i} \chi_{0}^{m-i} \chi_{1}^{i} y^{i-1} g^{-1} x^{m-i} .
$$

Comparing coefficients we obtain that for any $i=1 \ldots m-1$

$$
\frac{b}{a} \alpha_{i} \chi_{1}^{m-i}=\alpha_{i+1} \chi_{1}^{i+1}
$$

From this equation we obtain that $\alpha_{i}=\gamma \chi_{i}^{m} a^{m-i} b^{i}$, for some $\gamma \in \mathbb{k}$, thus $\theta=\gamma(a x+b y)^{m}$.

I. Heckenberger pointed out to me that the above result is a very special property that holds for quantum linear spaces, clearly not valid for arbitrary Nichols algebras.

Let us define collections of left $\mathcal{H}(1, q)$-comodule algebras right $\mathcal{H}(1, q)$ simple. The algebras in the list are pair-wise non isomorphic as comodule algebras. Let $d \in \mathbb{N}$ be a divisor of $n$ and $d m=n$.

(a) The group algebra $\mathbb{k} C_{d}=\mathbb{k}\left\langle h: h^{d}=1\right\rangle$, with coaction determined by $\lambda(h)=g^{m} \otimes h$.

(b) For any $\xi \in \mathbb{k}$, the algebras $\mathcal{A}_{0}(d, \xi)=\mathbb{k}\left\langle h, w: h^{d}=1, w^{n}=\right.$ $\left.\xi 1, h w=q^{m} w h\right\rangle$ and coaction determined by $\lambda(h)=g^{m} \otimes h, \lambda(w)=$ $x \otimes 1+g^{-1} \otimes w$.

(c) For any $\xi \in \mathbb{k}$, the algebras $\mathcal{A}_{1}(d, \xi)=\mathbb{k}\left\langle h, w: h^{d}=1, w^{n}=\right.$ $\left.\xi 1, h w=q^{m} w h\right\rangle$ and coaction determined by $\lambda(h)=g^{m} \otimes h, \lambda(w)=$ $y \otimes 1+g^{-1} \otimes w$.

(d) For any $\xi, \mu \in \mathbb{k}, \mu \neq 0$, the algebras $\mathcal{A}(\xi, \mu)=\mathbb{k}\left\langle w: w^{n}=\xi 1\right\rangle$ with coaction determined by $\lambda(w)=(\mu x+y) \otimes 1+g^{-1} \otimes w$.

(e) For any $\xi, \mu \in \mathbb{k}$, the algebras $\mathcal{D}(d, \xi, \mu)=\mathbb{k}\left\langle h, w, z: h^{d}=1, w^{n}=\right.$ $\left.\xi 1, z^{n}=\mu 1, h z=q^{m} z h, h w=q^{-m} w h, z w-q w z=0\right\rangle$. The coaction is determined by $\lambda(h)=g^{m} \otimes h, \lambda(z)=x \otimes 1+g^{-1} \otimes z$, $\lambda(w)=y \otimes 1+g^{-1} \otimes w$.

(f) For any $\xi, \mu, \eta \in \mathbb{k}$, the algebras $\mathcal{D}_{1}(\xi, \mu, \eta)=\mathbb{k}\left\langle h, w, z: h^{n}=\right.$ $1, w^{n}=\xi 1, z^{n}=\mu 1, h z=q z h, h w=q^{-1} w h, z w-q w z=$ 
$\left.\eta h^{n-2}\right\rangle$. The coaction is determined by $\lambda(h)=g \otimes h, \lambda(z)=x \otimes 1+$ $g^{-1} \otimes z, \lambda(w)=y \otimes 1+g^{-1} \otimes w$.

(g) Assume that $n=2 k, 1<k \in \mathbb{N}$. For any $\xi, \mu \in \mathbb{k}$, the algebras $\mathcal{D}_{2}(\xi, \mu, \eta)=\mathbb{k}\left\langle h, w, z: h^{k}=1, w^{n}=\xi 1, z^{n}=\mu 1, h z=\right.$ $\left.q^{2} z h, h w=q^{-2} w h, z w-q w z=\eta h^{k-1}\right\rangle$. The coaction is determined by $\lambda(h)=g^{2} \otimes h, \lambda(z)=x \otimes 1+g^{-1} \otimes z, \lambda(w)=y \otimes 1+g^{-1} \otimes w$.

Lemma 8.8. The algebras listed above are of the form ${ }_{\sigma} K$, where $K \subseteq$ $\mathcal{H}(1, q)$ is a left coideal subalgebra and $\sigma$ is a compatible Hopf 2-cocycle. Moreover the algebras listed above are pair-wise non Morita equivariant equivalent.

Proof. The proof is completely analogous to the proof of Lemma 8.5 .

Proposition 8.9. Let $\mathcal{M}$ be an exact indecomposable module category over $\operatorname{Rep}(\mathcal{H}(1, q))$, then $\mathcal{M} \simeq{ }_{K} \mathcal{M}$, where $K$ is one of the $H$-comodule algebras listed above.

Proof. Let $\left(G, \lambda_{0}\right)$ be a Loewy-graded $\mathcal{H}(1, q)$-comodule algebra such that $G_{0}=\mathbb{k} C_{d}$ for some divisor $d$ of $n$ and $G \neq G_{0}$. Follows from Proposition 7.5 that $G \simeq K \# G_{0}$ where $K \subseteq \mathfrak{B}(V)$ is an homogeneous left coideal subalgebra. Since $K(1) \neq 0$, follows from Lemma 8.7 that the only four possibilities for $K$ are the following. $K(1)=\mathbb{k}\langle x\rangle, K(1)=\mathbb{k}\langle y\rangle$, $K(1)=\mathbb{k}\langle\mu x+y\rangle$ for some $0 \neq \mu \in \mathbb{k}$, or $K=\mathfrak{B}(V)$. Notice that if $K(1)=\mathbb{k}<\mu x+y>$, since $K$ is invariant under the action of $G_{0}$ and $g \cdot x=q x, g \cdot y=q^{-1} y$ the only possibility is that $G_{0}=\mathbb{k} 1$, thus $d=1$.

Let $(A, \lambda)$ be a lifting of $G$ along $\mathcal{H}(1, q)$. In the first three cases it is easy to prove that $A \simeq \mathcal{A}_{0}(d, \xi), A \simeq \mathcal{A}_{1}(d, \xi)$ or $A \simeq \mathcal{A}(\xi, \mu)$ for some $\xi, \mu \in \mathbb{k}$.

Let us assume that $(A, \lambda)$ is a lifting of $\mathfrak{B}(V) \# \mathbb{k} C_{d}$ along $\mathcal{H}(1, q)$. Using the same arguments as in the proof of Proposition 8.3 one can prove that $A$ is generated by elements $h, w, z$ subject to relations $h^{d}=1, w^{n}=\xi 1, z^{n}=$ $\mu 1, h z=q^{m} z h, h w=q^{-m} w h$ for some $\xi, \mu \in \mathbb{k}$, and the coaction is determined by $\lambda(h)=g^{m} \otimes h, \lambda(z)=x \otimes 1+g^{-1} \otimes z, \lambda(w)=y \otimes 1+g^{-1} \otimes w$. Since $\lambda(z w-q w z)=g^{-2} \otimes(z w-q w z)$, then $z w-q w z \in A_{0}$. Thus there exists $\eta_{i} \in \mathbb{k}$ such that $z w-q w z=\sum_{i=0}^{d} \eta_{i} h^{i}$. Therefore

$$
\sum_{i=0}^{d} \eta_{i} g^{m i} \otimes h^{i}=\sum_{i=0}^{d} \eta_{i} g^{-2} \otimes h^{i} .
$$

We conclude that there are two possibilities: if there is no $i=0 \ldots d-1$ such that $g^{-2}=g^{m i}$, in which case all $\eta_{i}=0$ and $z w-q w z=0$, thus $A \simeq \mathcal{D}(d, \xi, \mu)$. The other case is when there exists one $i=0 \ldots d-1 \mathrm{such}$ that $g^{-2}=g^{m i}$ thus $\eta_{j}=0$ for all $j$ except when $j=i$. Also $n \mid m i+2$. Since $i<d, m i+2<n+2$ then $n=m i+2$, but since $m$ is a divisor of $n$, this implies that $m$ divides 2 , hence $m=1$ or $m=2$. In the first case $d=n$ and $A \simeq \mathcal{D}_{1}(\xi, \mu, \eta)$ and in the second case $A \simeq \mathcal{D}_{2}(\xi, \mu, \eta)$. 
8.4. Module categories over $\operatorname{Rep}\left(u_{q}\left(\mathfrak{s l}_{2}\right)\right)$. For convenience we shall work over an isomorphic Hopf algebra rather than with $u_{q}\left(\mathfrak{s l}_{2}\right)$ itself.

Let $2<n \in \mathbb{N}$ and $q \in \mathbb{k}$ be an $n$-th primitive root of 1 . Let $\mathcal{H}_{q}$ be the algebra generated by elements $g, x, y$ subject to relations

$$
g x=q^{2} x g, \quad g y=q^{-2} y g, \quad g^{n}=1, x^{n}=0, y^{n}=0, \quad x y-q^{2} y x=1-g^{-2} .
$$

The algebra $\mathcal{H}_{q}$ is a Hopf algebra with coproduct determined by $\Delta(g)=g \otimes g$, $\Delta(x)=x \otimes 1+g^{-1} \otimes x, \Delta(y)=y \otimes 1+g^{-1} \otimes y$.

It is easy to verify that $\mathcal{H}_{q} \simeq u_{q}\left(\mathfrak{s l}_{2}\right)$ as Hopf algebras, where the isomorphism is given by multiplying by the group-like element $g$ and that $\operatorname{gr}_{c} \mathcal{H}_{q} \simeq$ $\mathfrak{B}(V) \# \mathbb{k} C_{n}$ as Hopf algebras, where $V=\mathbb{k}\{x, y\}$ is the 2-dimensional Yetter-Drinfeld module over $\mathbb{k} C_{n}$ with structure maps $\delta: V \rightarrow \mathbb{k} C_{n} \otimes_{\mathbb{k}} V$, $\cdot: \mathbb{k} C_{n} \otimes_{\mathbb{k}} V \rightarrow V$ given by

$$
\delta(x)=g^{-1} \otimes x, \delta(y)=g^{-1} \otimes y, g \cdot x=q^{2} x, g \cdot y=q^{-2} y .
$$

Let us define a family of left $\mathcal{H}_{q}$-comodule algebras $\mathcal{H}_{q}$-simple. Let $d \in \mathbb{N}$ be a divisor of $n$ and $d m=n$.

(a) The group algebra $\mathbb{k} C_{d}=\mathbb{k}\left\langle h: h^{d}=1\right\rangle$, with coaction determined by $\lambda(h)=g^{m} \otimes h$.

(b) For any $\xi \in \mathbb{k}$, the algebras $\mathcal{A}_{0}(d, \xi)=\mathbb{k}\left\langle h, w: h^{d}=1, w^{n}=\right.$ $\left.\xi 1, h w=q^{m} w h\right\rangle$ and coaction determined by $\lambda(h)=g^{m} \otimes h, \lambda(w)=$ $x \otimes 1+g^{-1} \otimes w$.

(c) For any $\xi \in \mathbb{k}$, the algebras $\mathcal{A}_{1}(d, \xi)=\mathbb{k}\left\langle h, w: h^{d}=1, w^{n}=\right.$ $\left.\xi 1, h w=q^{m} w h\right\rangle$ and coaction determined by $\lambda(h)=g^{m} \otimes h, \lambda(w)=$ $y \otimes 1+g^{-1} \otimes w$.

(d) For any $\xi, \mu \in \mathbb{k}$, the algebras $\mathcal{B}(d, \xi, \mu)=\mathbb{k}\left\langle h, w, z: h^{d}=1, w^{n}=\right.$ $\left.\xi 1, \quad z^{n}=\xi 1, \quad h w=q^{m} w h, \quad h z=q^{m} z h, \quad z w-q^{2} w z=1\right\rangle$, with coaction determined by $\lambda(h)=g^{m} \otimes h, \lambda(w)=y \otimes 1+g^{-1} \otimes w$, $\lambda(z)=x \otimes 1+g^{-1} \otimes z$.

(e) If there exists $a \in \mathbb{N}$ such that $m a+2=n$, then for any $\xi, \mu, \eta \in \mathbb{k}$, with $\eta \neq 0$, set the algebras $\mathcal{C}(d, \xi, \mu, \eta, a)=\mathbb{k}\left\langle h, w, z: h^{d}=1, w^{n}=\right.$ $\left.\xi 1, z^{n}=\xi 1, h w=q^{m} w h, h z=q^{m} z h, z w-q^{2} w z=1+\eta h^{a}\right\rangle$, with coaction determined by $\lambda(h)=g^{m} \otimes h, \lambda(w)=y \otimes 1+g^{-1} \otimes w$, $\lambda(z)=x \otimes 1+g^{-1} \otimes z$.

Lemma 8.10. The algebras listed above are of the form ${ }_{\sigma} K$, where $K \subseteq \mathcal{H}_{q}$ is a left coideal subalgebra and $\sigma$ is a compatible 2-cocycle. Moreover the algebras listed above are pair-wise non Morita equivariant equivalent.

Proof. The proof is completely analogous to the proof of Lemma 8.5 .

Proposition 8.11. If $\mathcal{M}$ is an indecomposable exact module category over $\operatorname{Rep}\left(u_{q}\left(\mathfrak{s l}_{2}\right)\right)$ then $\mathcal{M}$ is equivalent to one of the following categories: $\mathbb{k}_{\mathbb{k}} \mathcal{M}$,

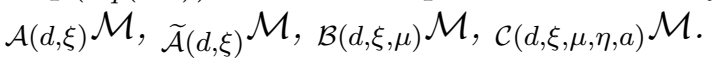


Proof. Let $\left(G, \lambda_{0}\right)$ be a Loewy-graded $\mathfrak{B}(V) \# \mathbb{k} C_{n}$-comodule algebra. We assume that $G \neq G_{0}$ and $G_{0}=\mathbb{k}\left\langle h: h^{d}=1\right\rangle$ for some $d$ divisor of $n$. The space $\mathfrak{B}_{G} \subseteq \mathfrak{B}(V)$ is an homogeneous left coideal subalgebra generated as an algebra by $\mathfrak{B}_{G}(1)$. This can be proven using the same arguments as in the proof of Lemma 8.7 .

Assume that $\operatorname{dim} \mathfrak{B}_{G}(1)=2$, then evidently $\mathfrak{B}_{G}=\mathfrak{B}(V)$. Let $(A, \lambda)$ be a lifting of $\left(G, \lambda_{0}\right)$ along $\mathcal{H}_{q}$. Arguing as in Proposition 8.9 we obtain that $A$ is generated by elements $h, w, z$ subject to relations

$$
h^{d}=1, \quad w^{n}=\xi 1, \quad z^{n}=\xi 1, \quad h w=q^{m} w h, \quad h z=q^{m} z h .
$$

Where the coaction is determined by $\lambda(h)=g^{m} \otimes h, \lambda(z)=x \otimes 1+g^{-1} \otimes z$, $\lambda(w)=y \otimes 1+g^{-1} \otimes w$. Since

$$
\lambda\left(z w-q^{2} w z\right)=\left(1-g^{-2}\right) \otimes 1+g^{-2} \otimes\left(z w-q^{2} w z\right),
$$

then there exists $\alpha_{i} \in \mathbb{k}, i=0 \ldots d-1$ such that $z w-q^{2} w z=\sum_{i} \alpha_{i} h^{i}$. Using equation (8.2) we obtain that

$$
\sum_{i} \alpha_{i} g^{m i} \otimes h^{i}=\left(1-g^{-2}\right) \otimes 1+\sum_{i} \alpha_{i} g^{-2} \otimes h^{i} .
$$

This implies that $\alpha_{0}=1$. If there is no $1 \leq i \leq d-1$ such that $m i=n-2$ then $\alpha_{i}=0$ for all $i=1 \ldots d-1$ and in this case $A \simeq \mathcal{B}(d, \xi, \mu)$. If there is a natural number $a$ such that $m a=n-2$ then $\alpha_{i}=0$ for all $i \neq a$ and $\alpha_{a}$ is arbitrary. In this case $A \simeq \mathcal{C}\left(d, \xi, \mu, \alpha_{a}, a\right)$.

If $\operatorname{dim} \mathfrak{B}_{G}(1)=1$, we have three possibilities: $\mathfrak{B}_{G}(1)=\mathbb{k}\langle x\rangle, \mathfrak{B}_{G}(1)=$ $\mathbb{k}<y>$ or $\mathfrak{B}_{G}(1)=\mathbb{k}<\mu x+y>$ for some $\mu \in \mathbb{k}^{\times}$. In the firs two cases $A \simeq \mathcal{A}_{0}(d, \xi)$ or $A \simeq \mathcal{A}_{1}(d, \xi)$ respectively. Let us prove that there is no lifting in the third case. Our proof relies on the fact that $(\mu x+y)^{n} \in \mathcal{H}_{q}$ is a non-zero element. If there is a lifting $(A, \lambda)$ of $\left(G, \lambda_{0}\right)$ then there exists an element $z \in A$ such that $\lambda(z)=(\mu x+y) \otimes 1+g^{-1} \otimes z$ therefore

$$
\lambda\left(z^{n}\right)=(\mu x+y)^{n} \otimes 1+1 \otimes z^{n} .
$$

The coassociativity of $\lambda$ implies that the element $(\mu x+y)^{n}$ must be a non-zero primitive element of $\mathcal{H}_{q}$, which is impossible since $\mathcal{H}_{q}$ is finitedimensional, see [Sw69, Thm. 13.0.1].

\section{Some Further COMments}

1. Notice that if $H$ is one of the Hopf algebras in the following list:

(i) a group algebra $\mathbb{k} G$ of a finite group $G$,

(ii) a Taft Hopf algebra $T_{q}$,

(iii) a Radford Hopf algebra $\mathbf{r}(q)$,

(iv) the Frobenius-Lusztig kernel $u_{q}\left(\mathfrak{s l}_{2}\right)$,

then exact module categories over $\operatorname{Rep}(H)$ are of the form ${ }_{\sigma K} \mathcal{M}$, where $K \subseteq H$ is a left coideal subalgebra and $\sigma$ is a compatible Hopf 2-cocycle. 
It would be interesting to know if this statement is true for any finitedimensional pointed Hopf algebra with abelian coradical.

2. Using the same strategy as for the book Hopf algebras and for the Radford Hopf algebras it is likely that a classification for a bosonization of quantum linear spaces can be worked out easily. Also the results by Etingof and Ostrik on the classification of exact module categories over finite supergroups $\wedge V \# \mathbb{k} G$, see [EO04, section 4.2], can be obatined using our techniques, at least when $G$ is a cyclic group.

3. In principle the techniques developed here would help to classify exact module categories over $\operatorname{Rep}\left(u_{q}\left(\mathfrak{s l}_{n}\right)\right)$, since in the paper [KLS08] the authors classified all homogeneous coideal subalgebras of $u_{q}\left(\mathfrak{s l}_{n}\right)$.

Acknowledgments. I am grateful for the kind hospitality at LMU, specially many thanks to Professor H.-J. Schneider. I also want to mention Professor I. Heckenberger for sharing his insights on Nichols algebras.

\section{REFERENCES}

[AM07] N. Andruskiewitsch and J.M. Mombelli, On module categories over finitedimensional Hopf algebras, J. Algebra 314 (2007), 383-418.

[AS98] N. ANDruskiewitsch and H.-J. Schneider, Lifting of quantum linear spaces and pointed Hopf algebras of order $p^{3}$, J. Algebra 209 (1998), 658-691.

[AS02] N. Andruskiewitsch and H.-J. Schneider, Pointed Hopf Algebras, in New directions in Hopf algebras, 1-68, Math. Sci. Res. Inst. Publ. 43, Cambridge Univ. Press, Cambridge, 2002.

[AD03] N. Andruskiewitsch and S. DăsCăLESCU, Co-Frobenius Hopf algebras and the coradical filtration, Math. Z. 243, 145154 (2003)

[Be67] BÉnabou, J., Introduction to bicategories, Reports of the Midwest Category Seminar (1967), Lecture Notes in Math. 47, pp. 1-77, Springer, Berlin.

[BEK00] J. Böckenhauer, D. E. Evans and Y. Kawahigashi, Chiral Structure of Modular Invariants for Subfactors, CMP 210 (2000), 733-784.

[BO01] R. BezRukAVNikov and V. Ostrik, On tensor categories attached to cells in affine Weyl groups II, math.RT/0102220.

[DCY05] Y. DU, X.-W. CHEN, and Y. YE, On graded bialgebra deformations, math.QA/0506030.

[CS07] R. CoquereauX and G. Schieber, Orders and dimensions for $\mathfrak{s l}_{2}$ and $\mathfrak{s l}_{3}$ module categories and boundary conformal field theories on a torus, J. of Mathematical Physics, 48, 043511 (2007).

[CS08] R. Coquereaux and G. Schieber, From conformal embeddings to quantum symmetries: an exceptional SU(4) example, Journal of Physics- Conference Series Volume 103 (2008), 012006.

[EO04] P. Etingof and V. Ostrik, Finite tensor categories, Mosc. Math. J. 4 (2004), no. 3, 627-654.

[EnO05] P. Etingof, D. Nikshych and V. Ostrik, On fusion categories, Ann. Math. 162, 581-642 (2005).

[ENO08] P. Etingof, D. Nikshych and V. Ostrik, Weakly group-theoretical and solvable fusion categories, preprint arXiv:0809.3031.

[FS01] J. Fuchs and C. SChWEIGERT, Category theory for conformal boundary conditions, in Vertex Operator Algebras in Mathematics and Physics, (2000). Preprint math.CT/0106050. 
[KLS08] V.K. Kharchenko and A.V. Lara Sagahon, Right coideal subalgebras in $U_{q}\left(\mathfrak{s l}_{n+1}\right)$, J. Algebra 319 (2008) 2571-2625.

[KO02] A. Kirillov JR. and V. OSTRIK, On a q-analogue of the McKay correspondence and the ADE classification of sl $_{2}$ conformal field theories, Adv. Math. 171 (2002), no. 2, 183-227.

[Li03] V. Linchenko, Nilpotent subsets of Hopf module algebras, in Groups, Rings, Lie and Hopf Algebras, Y. Bahturin (Ed.) (2003).

[Ma92] A. MASuOKA, Freeness of Hopf algebras over coideal subalgebras, Comm. in Algebra 20 5, 1353-1373 (1992).

[M07] M. Mombelli, Dynamical twists in Hopf algebras, Int. Math. Res. Not. (2007) vol.2007.

[MW07] M. MASTNAK and S. Witherspoon, Bialgebra cohomology, pointed Hopf algebras, and deformations, preprint math.RA/0704.2771.

[N07] D. NiKSHYCH, Non group-theoretical semisimple Hopf algebras from group actions on fusion categories, preprint arXiv:0712.0585.

[O03a] V. Ostrik, Module categories, Weak Hopf Algebras and Modular invariants, Transform. Groups, 2 8, 177-206 (2003).

[O03b] V. Ostrik, Module categories over the Drinfeld double of a Finite Group, Int. Math. Res. Not. 2003, no. 27, 1507-1520.

[O08] V. Ostrik, Module Categories Over Representations of $S L_{q}(2)$ in the NonSemisimple Case, Geom. funct. anal. Vol. 17 (2008), 2005-2017.

[Sk07] S. Skryabin, Projectivity and freeness over comodule algebras, Trans. Am. Math. Soc. 359, No. 6, 2597-2623 (2007).

[SS04] H-J. Schneider and P. Schauenburg, Galois type extensions and Hopf algebras, preprint (2004), 89 pages.

[Sw69] M. SweEdLer, Hopf algebras, Benjamin, New York, 1969.

[Y07] D. YAU, Cohomology and deformation of module-algebras, J. Pure Appl. Algebra 209 (2007), 781-792.

[Y08] D. YAU, Deformation bicomplex of module-algebras, Homology Homotopy Appl. 10, Number 1 (2008), 97-128.

Mathematisches Institut, Universität München, Theresienstrasse 39, D-80333 München, Germany.

E-mail address: mombelli@mate.uncor.edu, martin10090@gmail.com

URL: http://www.mate.uncor.edu/mombelli 